\title{
DISCRIMINANTS OF BRAUER ALGEBRAS
}

\author{
HEBING RUI AND MEI SI
}

\begin{abstract}
In this paper, we compute Gram determinants associated to all cell modules of Brauer algebras $\mathscr{B}_{n}(\delta)$. Theoretically, we know when a cell module of $\mathscr{B}_{n}(\delta)$ is equal to its simple head. This gives a solution of this long standing problem.
\end{abstract}

On the occasion of Professor Gus Lehrer's 60 ${ }^{\text {th }}$ birthday

\section{INTRODUCTION}

In [Bra37], Richard Brauer introduced a class of finite dimensional algebras $\mathscr{B}_{n}(\delta)$ in order to study the $n$-th tensor power of the defining representations of orthogonal groups and symplectic groups. Such algebras, which are known as Brauer algebras or Brauer centraliser algebras, have been studied by many authors; see for example BW89, Bro56, DWH99, Eny04, Eny05, FG95, HR95, HW89a, HW89b, Jon94, LR97, Naz96, Ram95, Rui05, RS06a, Ter01,Wen88, Wey97, etc.

In [GL96], Graham and Lehrer introduced the notion of cellular algebra which is defined over a poset $\Lambda$. Such an algebra has a nice basis, called a cellular basis. For each $\lambda \in \Lambda$, there is a module $\Delta(\lambda)$ called a cell module. Graham and Lehrer showed that there is a symmetric, associative bilinear form $\phi_{\lambda}$ defined on $\Delta(\lambda)$. It has been proved in [GL96, 3.8] that a cellular algebra is (split) semisimple if and only if $\phi_{\lambda}$ is non-degenerate for any $\lambda \in \Lambda$.

Graham and Lehrer proved that $\mathscr{B}_{n}(\delta)$ over a commutative ring is a cellular algebra over the poset $\Lambda$ which consists of all pairs $(f, \lambda)$, with $0 \leq f \leq\lfloor n / 2\rfloor$ and $\lambda$ being a partition of $n-2 f$. Here $\lfloor n / 2\rfloor$ is the maximal integer with $\lfloor n / 2\rfloor \leq n / 2$. Therefore, $\mathscr{B}_{n}(\delta)$ is (split) semisimple over a field if and only if $\phi_{f, \lambda}$ are non-degenerate for all $(f, \lambda) \in \Lambda$.

In [Rui05], the first author proved that $\mathscr{B}_{n}(\delta)$ with $\delta \neq 0$ is semisimple if and only if (a) the group algebra of the symmetric group $\mathfrak{S}_{n}$ is semisimple, (b) $\phi_{1, \lambda}$ are non-degenerate for all partitions $\lambda$ of $k-2$ and $2 \leq k \leq n$. By [DWH99, 3.3-3.4], he gave an algorithm to determine whether $\phi_{1, \lambda}$ is non-degenerate or not. When $\delta=0$, the semisimplicity of $\mathscr{B}_{n}(\delta)$ can be determined by direct computation [Rui05. In [RS06a], we used such an algorithm to verify Enyang's conjecture in [Eny05, which is about the semisimplicity of Brauer algebras. This gives a complete solution of the problem of semisimplicity of $\mathscr{B}_{n}(\delta)$ over an arbitrary field. In other words we have

The first author is supported in part by NSFC and NCET. 
found all $\delta$ such that no Gram determinant associated to a cell module is equal to zero.

A further question is when the Gram determinant for a fixed cell module is not equal to zero. This is equivalent to finding a necessary and sufficient condition for a cell module of $\mathscr{B}_{n}(\delta)$ being equal to its simple head.

The main purpose of this paper is to solve the above problem. In [Eny05], Enyang constructed the Jucys-Murphy basis of each cell module for $\mathscr{B}_{n}(\delta)$. In this paper, we will use his result to construct the Jucys-Murphy basis for $\mathscr{B}_{n}(\delta)$ over a commutative ring. Since the Jucys-Murphy elements of $\mathscr{B}_{n}(\delta)$, which were defined by Nazarov in [Naz96], act on the Jucys-Murphy basis of $\mathscr{B}_{n}(\delta)$ via upper triangular matrices, we can and do use some arguments in [JM00] or Mat04 to construct an orthogonal basis of $\mathscr{B}_{n}(\delta)$ under the assumption 3.1. We show that the Gram determinants associated to each cell module, which are defined via the Jucys-Murphy basis and this orthogonal basis, are same. Using classical branching rule for $\mathscr{B}_{n}(\delta)$, we will compute each diagonal entry of the Gram matrix defined via this orthogonal basis. This gives a recursive formula for each Gram determinant. We remark that such recursive formulae shed some light on possible composition factors of cell modules when they are not simple. Also, we will use such recursive formulae to describe (up to a sign) the actions of generators of $\mathscr{B}_{n}(\delta)$ on each orthogonal basis element. As an application, we construct explicitly primitive idempotents and central primitive idempotents of $\mathscr{B}_{n}(\delta)$. This gives the Wedderburn-Artin decomposition of Brauer algebras $\mathscr{B}_{n}(\delta)$ under the assumption 3.1 .

Finally, we remark that the method in this paper will be used to deal with Birman-Murakami-Wenzl algebras [BW89] and cyclotomic Nazarov-Wenzl algebras AMR06]. Details will appear elsewhere.

Acknowledgement: The first author thanks Prof. A. Mathas for inviting him to the University of Sydney in January 2005, and Prof. G. Lehrer for suggesting to investigate discriminants for Brauer algebras. We thank A. Mathas for his comments on the paper. Finally, both of us thank the referee for their valuable comments.

\section{Brauer ALGEBras}

Throughout, let $R$ be a commutative ring which contains the identity 1 and $\delta$. The Brauer algebra $\mathscr{B}_{n}(\delta)$ is a unital associative $R$-algebra generated by $s_{i}, e_{i}, 1 \leq i \leq n-1$, subject to the following relations:

$$
\begin{aligned}
& s_{i}^{2}=1 \text {, } \\
& e_{i}^{2}=\delta e_{i}, \\
& s_{i} e_{i}=e_{i} s_{i}=e_{i}, \\
& s_{i} s_{j}=s_{j} s_{i}, \\
& s_{i} e_{j}=e_{j} s_{i}, \\
& e_{i} e_{j}=e_{j} e_{i}, \\
& s_{k} s_{k+1} s_{k}=s_{k+1} s_{k} s_{k+1} \text {, } \\
& e_{k} e_{k+1} e_{k}=e_{k}, \\
& e_{k+1} e_{k} e_{k+1}=e_{k+1} \text {, } \\
& s_{k} e_{k+1} e_{k}=s_{k+1} e_{k}, \\
& e_{k+1} e_{k} s_{k+1}=e_{k+1} s_{k},
\end{aligned}
$$


where $1 \leq i, j<n$, with $|i-j|>1$, and $1 \leq k<n-1$.

Let $\operatorname{Sym}(T)$ be the symmetric group on the set $T$. If $T=\{1,2, \cdots, n\}$, we denote $\operatorname{Sym}(T)$ by $\mathfrak{S}_{n}$. The group algebra $R \mathfrak{S}_{n}$ can be considered as a subalgebra of $\mathscr{B}_{n}(\delta)$ if we identify $s_{i}$ with the basic transposition $(i, i+1)$.

We are going to construct the Jucys-Murphy basis of $\mathscr{B}_{n}(\delta)$. We start by recalling some combinatorics.

Recall that a partition of $n$ is a weakly decreasing sequence of nonnegative integers $\lambda=\left(\lambda_{1}, \lambda_{2}, \ldots\right)$ such that $|\lambda|:=\lambda_{1}+\lambda_{2}+\cdots=n$. In this situation, we write $\lambda \vdash n$. Let $\Lambda^{+}(n)$ be the set of all partitions of $n$. It is well known that $\Lambda^{+}(n)$ is a poset with dominance order $\unlhd$ as the partial order on it. Given $\lambda, \mu \in \Lambda^{+}(n), \lambda \unlhd \mu$ if $\sum_{j=1}^{i} \lambda_{j} \leq \sum_{j=1}^{i} \mu_{j}$ for all possible $i$. Write $\lambda \triangleleft \mu$ if $\lambda \unlhd \mu$ and $\lambda \neq \mu$.

Suppose that $\lambda$ and $\mu$ are two partitions. We say that $\mu$ is obtained from $\lambda$ by adding a box if there exists an $i$ such that $\mu_{i}=\lambda_{i}+1$ and $\mu_{j}=\lambda_{j}$ for all $j \neq i$. In this situation we will also say that $\lambda$ is obtained from $\mu$ by removing a box and we write $\lambda \rightarrow \mu$ and $\mu \backslash \lambda=\left(i, \mu_{i}\right)$. We will also say that the pair $\left(i, \mu_{i}\right)$ is an addable node of $\lambda$ and a removable node of $\mu$. Note that $|\mu|=|\lambda|+1$.

The Young diagram $Y(\lambda)$ for a partition $\lambda=\left(\lambda_{1}, \lambda_{2}, \cdots\right)$ is a collection of boxes arranged in left-justified rows with $\lambda_{i}$ boxes in the $i$-th row of $Y(\lambda)$. A $\lambda$-tableau $\mathbf{s}$ is obtained by inserting $i, 1 \leq i \leq n$ into $Y(\lambda)$ without repetition. $\mathfrak{S}_{n}$ acts on $\mathbf{s}$ by permuting its entries. Let $\mathbf{t}^{\lambda}$ be the $\lambda$-tableau obtained from $Y(\lambda)$ by adding $1,2, \cdots, n$ from left to right along the rows of $Y(\lambda)$. If $\mathbf{t}^{\lambda} w=\mathbf{s}$, write $w=d(\mathbf{s})$. Note that $d(\mathbf{s})$ is uniquely determined by $\mathbf{s}$.

A $\lambda$-tableau $\mathbf{s}$ is standard if the entries in $\mathbf{s}$ increase both from left to right in each row and from top to bottom in each column. Let $\mathscr{T}_{n}^{\text {std }}(\lambda)$ be the set of all standard $\lambda$-tableaux.

Given an $\mathbf{s} \in \mathscr{T}_{n}^{\text {std }}(\lambda)$, let $\mathbf{s} \downarrow_{i}$ be obtained from $\mathbf{s}$ by removing all boxes containing the entries $j$ in $\mathbf{s}$ with $j>i$. Let $\mathbf{s}_{i}$ denote the partition of $i$ such that $\mathbf{s}_{i}$ is an $\mathbf{s}_{i}$-tableau. Then $\mathbf{s}=\left(\mathbf{s}_{0}, \mathbf{s}_{1}, \cdots, \mathbf{s}_{n}\right)$ is a sequence of partitions such that $\mathbf{s}_{i} \rightarrow \mathbf{s}_{i+1}$. Conversely, if we insert $i$ into $\mathbf{s}_{i} \backslash \mathbf{s}_{i-1}$, we will obtain an $\mathbf{s} \in \mathscr{T}_{n}^{\text {std }}(\lambda)$. Therefore, there is a bijection between $\mathscr{T}_{n}^{\text {std }}(\lambda)$ and the set of all $\left(\mathbf{s}_{0}, \mathbf{s}_{1}, \cdots, \mathbf{s}_{n}\right)$ with $\mathbf{s}_{0}=\varnothing, \mathbf{s}_{n}=\lambda$ and $\mathbf{s}_{i} \rightarrow \mathbf{s}_{i+1}, 0 \leq i \leq n-1$.

Assume that $0 \leq f \leq\left\lfloor\frac{n}{2}\right\rfloor$. Let $\mathfrak{S}_{n-2 f}$ be the symmetric group on $\{2 f+$ $1,2 f+2, \cdots, n\}$. Following Eny05, let $\mathfrak{B}_{f}$ be the subgroup of $\mathfrak{S}_{n}$ generated by $\tilde{s}_{i}, \tilde{s}_{0}$, where $\tilde{s}_{i}=s_{2 i} s_{2 i-1} s_{2 i+1} s_{2 i}, 1 \leq i \leq f-1$, and $\tilde{s}_{0}=s_{1}$. In Eny05, Enyang proved that $\mathcal{D}_{f, n}$ is a complete set of right coset representatives of $\mathfrak{B}_{f} \times \mathfrak{S}_{n-2 f}$ in $\mathfrak{S}_{n}$, where

$\mathcal{D}_{f, n}=\left\{\begin{array}{l|l}w \in \mathfrak{S}_{n} \mid \begin{array}{l}(2 i+1) w<(2 j+1) w,(2 i+1) w<(2 i+2) w \text { for all } \\ 0 \leq i<j<f, \text { and }(k) w<(k+1) w \text { for } 2 f<k<n\end{array}\end{array}\right\}$. 
For $\lambda \vdash n-2 f$, let $\mathfrak{S}_{\lambda}$ be the Young subgroup of $\mathfrak{S}_{n-2 f}$ generated by $s_{j}$, $2 f+1 \leq j \leq n-1$ and $j \neq 2 f+\sum_{k=1}^{i} \lambda_{k}$ for all possible $i$. A standard $\lambda$ tableau $\hat{\mathbf{s}}$ is obtained by using $2 f+i, 1 \leq i \leq n-2 f$ instead of $i$ in the usual standard $\lambda$-tableau s. Define $d(\hat{\mathbf{s}}) \in \mathfrak{S}_{n-2 f}$ by declaring that $\hat{\mathbf{s}}=\hat{\mathbf{t}}^{\lambda} d(\hat{\mathbf{s}})$. If there is no confusion, we will use $\mathbf{s}$ instead of $\hat{\mathbf{s}}$.

In [GL96], Graham and Lehrer proved that $\mathscr{B}_{n}(\delta)$ is a cellular algebra over a commutative ring $R$. In the current paper, we are going to recall Enyang's cellular basis for $\mathscr{B}_{n}(\delta)$. We start by recalling the notion of cellular algebra.

Definition 2.1 ( GL96]). Let $R$ be a commutative ring and $A$ an $R$ algebra. Fix a partially ordered set $\Lambda=(\Lambda, \unrhd)$ and for each $\lambda \in \Lambda$ let $T(\lambda)$ be a finite set. Finally, fix $C_{\mathfrak{s t}}^{\lambda} \in A$ for all $\lambda \in \Lambda$ and $\mathfrak{s}, \mathfrak{t} \in T(\lambda)$.

Then the triple $(\Lambda, T, C)$ is a cell datum for $A$ if:

a) $\left\{C_{\mathfrak{s t}}^{\lambda} \mid \lambda \in \Lambda\right.$ and $\left.\mathfrak{s}, \mathfrak{t} \in T(\lambda)\right\}$ is an $R$-basis for $A$;

b) the $R$-linear map $*: A \longrightarrow A$ determined by $\left(C_{\mathfrak{s t}}^{\lambda}\right)^{*}=C_{\mathfrak{t}}^{\lambda}$, for all $\lambda \in \Lambda$ and all $\mathfrak{s}, \mathfrak{t} \in T(\lambda)$ is an anti-isomorphism of $A$;

c) for all $\lambda \in \Lambda, \mathfrak{s} \in T(\lambda)$ and $a \in A$ there exist scalars $r_{\mathfrak{s u}}(a) \in R$, which are independent of $\mathfrak{t}$, such that

$$
a C_{\mathfrak{s t}}^{\lambda}=\sum_{\mathfrak{u} \in T(\lambda)} r_{\mathfrak{s u}}(a) C_{\mathfrak{u t}}^{\lambda} \quad\left(\bmod A^{\triangleright \lambda}\right),
$$

where $A^{\triangleright \lambda}=R$-span $\left\{C_{\mathfrak{u v}}^{\mu} \mid \mu \triangleright \lambda\right.$ and $\left.\mathfrak{u}, \mathfrak{v} \in T(\mu)\right\}$.

An algebra $A$ is a cellular algebra if it has a cell datum and in this case we call $\left\{C_{\mathfrak{s t}}^{\lambda} \mid \mathfrak{s}, \mathfrak{t} \in T(\lambda), \lambda \in \Lambda\right\}$ a cellular basis of $A$. By (c), $A^{\triangleright \lambda}$ is a two-sided ideal of $A$.

Let $\Lambda_{n}=\left\{(f, \lambda) \mid \lambda \vdash n-2 f, 0 \leq f \leq\left\lfloor\frac{n}{2}\right\rfloor\right\}$. Given $(k, \lambda),(f, \mu) \in \Lambda_{n}$, define $(k, \lambda) \unlhd(f, \mu)$ if either $k<f$ or $k=f$ and $\lambda \unlhd \mu$. Write $(k, \lambda) \triangleleft(f, \mu)$, if $(k, \lambda) \unlhd(f, \mu)$ and $(k, \lambda) \neq(f, \mu)$.

Let $I(f, \lambda)=\mathscr{T}_{n}^{\text {std }}(\lambda) \times \mathcal{D}_{f, n}$. Write $m_{\lambda}=e^{f} x_{\lambda}$, where $e^{f}=e_{1} e_{3} \cdots e_{2 f-1}$ and $x_{\lambda}=\sum_{w \in \mathfrak{S}_{\lambda}} w$. Denote by

$$
C_{(\mathbf{s}, u)(\mathbf{t}, v)}^{(f, \lambda)}=u^{-1} d(\mathbf{s})^{-1} \mathfrak{m}_{\lambda} d(\mathbf{t}) v, \text { for all }(\mathbf{s}, u),(\mathbf{t}, v) \in I(f, \lambda) .
$$

Theorem 2.2. Eny04 Let $\mathscr{B}_{n}(\delta)$ be a Brauer algebra over a commutative ring $R$. Let $\sigma: \mathscr{B}_{n}(\delta) \rightarrow \mathscr{B}_{n}(\delta)$ be the $R$-linear involution which fixes $s_{i}, e_{j}$ for all $1 \leq i, j \leq n-1$. Then

a) $\mathscr{C}_{n}=\left\{C_{(\mathbf{s}, u)(\mathbf{t}, v)}^{(f, \lambda)} \mid(\mathbf{s}, u),(\mathbf{t}, v) \in I(f, \lambda), \lambda \vdash n-2 f, 0 \leq f \leq\left\lfloor\frac{n}{2}\right\rfloor\right\}$ is a free $R$-basis of $\mathscr{B}_{n}(\delta)$.

b) $\sigma\left(C_{(\mathbf{s}, u)(\mathbf{t}, v)}^{(f, \lambda)}\right)=C_{(\mathbf{t}, v)(\mathbf{s}, u)}^{(f, \lambda)}$ for all $(\mathbf{s}, u),(\mathbf{t}, v) \in I(f, \lambda)$ and all $(f, \lambda) \in$ $\Lambda_{n}$.

c) For all $h \in \mathscr{B}_{n}(\delta)$, and all $(\mathbf{s}, u),(\mathbf{t}, v) \in I(f, \lambda)$ with $(f, \lambda) \in \Lambda_{n}$,

$$
C_{(\mathbf{s}, u)(\mathbf{t}, v)}^{(f, \lambda)} h \equiv \sum_{(\mathbf{u}, w) \in I(f, \lambda)} a_{\mathbf{u}, w} C_{(\mathbf{s}, u)(\mathbf{u}, w)}^{(f, \lambda)}\left(\bmod \mathscr{B}_{n}(\delta)^{\triangleright(f, \lambda)}\right),
$$


where $\mathscr{B}_{n}(\delta)^{\triangleright(f, \lambda)}$ is the free $R$-submodule generated by $C_{(\tilde{\mathfrak{s}}, \tilde{u})(\tilde{\mathbf{t}}, \tilde{v})}^{(k, \mu)}$ with $(k, \mu) \triangleright(f, \lambda)$ and $(\tilde{\mathbf{s}}, \tilde{u}),(\tilde{\mathbf{t}}, \tilde{v}) \in I(k, \mu)$. Moreover, each coefficient $a_{\mathbf{u}, w}$ is independent of $(\mathbf{s}, u)$.

From here on, all modules considered in this paper are right modules.

For each $\lambda \vdash n-2 f$, Enyang considered the right module

$$
S^{\lambda}=R-\operatorname{span}\left\{\mathfrak{m}_{\lambda} d(\mathbf{t}) v \quad\left(\bmod \mathscr{B}_{n}(\delta)^{\triangleright(f, \lambda)}\right) \mid(\mathbf{t}, v) \in I(f, \lambda)\right\}
$$

By the definition of a cell module in [GL96], $S^{\lambda}$ is isomorphic to the cell module $\Delta(f, \lambda)$ with respect to the cellular basis $\mathscr{C}_{n}$, provided by Theorem 2.2. We will identify $\Delta(f, \lambda)$ with $S^{\lambda}$. We remark that the action of $\mathscr{B}_{n}(\delta)$ on $\Delta(f, \lambda)$ is given by Theorem 2.2 (c).

Suppose that $(f, \lambda) \in \Lambda_{n}$. An $n$-updown $\lambda$-tableau, or more simply an updown $\lambda$-tableau, is a sequence $\mathfrak{t}=\left(\mathfrak{t}_{0}, \mathfrak{t}_{1}, \mathfrak{t}_{2}, \ldots, \mathfrak{t}_{n}\right)$ of partitions, where $\mathfrak{t}_{n}=\lambda, \mathfrak{t}_{0}=\varnothing$, and the partition $\mathfrak{t}_{i}$ is obtained from $\mathfrak{t}_{i-1}$ by either adding or removing a box, for $i=1, \ldots, n$. Let $\mathscr{T}_{n}^{u d}(\lambda)$ be the set of all $n$-updown $\lambda$-tableaux. If $\lambda \vdash n$, an $n$-updown tableau becomes an $n$-up (i.e. there are no downs) tableau. In this case, there is a bijection between $\mathscr{T}_{n}^{u d}(\lambda)$ and $\mathscr{T}_{n}^{s t d}(\lambda)$.

For any $1 \leq i, j \leq n$, let

$$
s_{i, j}= \begin{cases}s_{i} s_{i+1} \cdots s_{j-1}, & \text { if } j>i \\ s_{i-1} s_{i-2} \cdots s_{j}, & \text { if } j<i \\ 1, & \text { if } i=j .\end{cases}
$$

Definition 2.3. Given $\mathfrak{t} \in \mathscr{T}_{n}^{u d}(\lambda)$ with $(f, \lambda) \in \Lambda_{n}$, define $f_{j} \in \mathbb{N}$ by declaring that $\mathfrak{t}_{j} \vdash j-2 f_{j}$. Let $\mu^{(j)}=\mathfrak{t}_{j}$. Following Eny05, define $\mathfrak{m}_{\mathfrak{t}}=\mathfrak{m}_{\mathfrak{t}_{n}}$ inductively by declaring that

(1) $\mathfrak{m}_{\mathfrak{t}_{1}}=1$,

(2) $\mathfrak{m}_{\mathfrak{t}_{i}}=\sum_{j=a_{k-1}+1}^{a_{k}} s_{j, i} \mathfrak{m}_{\mathfrak{t}_{i-1}}$ if $\mathfrak{t}_{i}=\mathfrak{t}_{i-1} \cup p$ with $p=\left(k, \mu_{k}^{(i)}\right)$, and $a_{l}=2 f_{i}+\sum_{j=1}^{l} \mu_{j}^{(i)}$

(3) $\mathfrak{m}_{\mathfrak{t}_{i}}=e_{2 f_{i}-1} s_{2 f_{i}, i} s_{2 f_{i}-1, b_{k}} \mathfrak{m}_{\mathfrak{t}_{i-1}}$ if $\mathfrak{t}_{i-1}=\mathfrak{t}_{i} \cup p$ with $p=\left(k, \mu_{k}^{(i-1)}\right)$, and $b_{k}=2\left(f_{i}-1\right)+\sum_{j=1}^{k} \mu_{j}^{(i-1)}$.

In Eny05, Enyang showed that $\mathfrak{m}_{\mathfrak{t}}=\mathfrak{m}_{\lambda} b_{\mathfrak{t}}$ for some $b_{\mathfrak{t}} \in R \mathfrak{S}_{n}$. The following recursive formulae describe explicitly $b_{\mathfrak{t}}$. Note that $b_{\mathfrak{t}}=b_{\mathfrak{t}_{n}}$ and $\mathfrak{t}_{n-1}=\mu$.

$$
b_{\mathfrak{t}_{n}}= \begin{cases}s_{a_{k}, n} b_{\mathfrak{t}_{n-1}}, & \text { if } \mathfrak{t}_{n}=\mathfrak{t}_{n-1} \cup\left\{\left(k, \lambda_{k}\right)\right\} \\ s_{2 f, n} \sum_{j=b_{k-1}+1}^{b_{k}} s_{2 f-1, j} b_{\mathfrak{t}_{n-1}}, & \text { if } \mathfrak{t}_{n-1}=\mathfrak{t}_{n} \cup\left\{\left(k, \mu_{k}\right)\right\} .\end{cases}
$$

For any $(f, \lambda) \in \Lambda_{n}$, define

- $\mathfrak{t}_{2 i-1}=(1)$ and $\mathfrak{t}_{2 i}=\varnothing$ for $1 \leq i \leq f$,

- $\mathfrak{t}_{i}$ is obtained from $\hat{\mathbf{t}}^{\lambda}$ by removing the entries $j$ with $j>i$ under the assumption $2 f+1 \leq i \leq n$. 
Then $\mathfrak{t}=\left(\mathfrak{t}_{0}, \mathfrak{t}_{1}, \cdots, \mathfrak{t}_{n}\right) \in \mathscr{T}_{n}^{u d}(\lambda)$. In this case, we denote $\mathfrak{t}$ by $\mathfrak{t}^{\lambda}$. By definition, $\mathfrak{m}_{\mathfrak{t}}=\mathfrak{m}_{\lambda}=e^{f} x_{\lambda}$. We remark that we use $\mathfrak{t}$ (resp. $\mathbf{t}$ ) to denote an updown (resp. a standard) tableau.

Following Eny05, let $\left(k_{i}, \mu^{(i)}\right) \in \Lambda_{n-1}, 1 \leq i \leq m$ be such that

a) $\mu^{(i)} \rightarrow \lambda$ if $k_{i}=f$,

b) $\lambda \rightarrow \mu^{(i)}$ if $k_{i}=f-1$,

c) $\left(k_{1}, \mu^{(1)}\right) \triangleright\left(k_{2}, \mu^{(2)}\right) \triangleright \cdots \triangleright\left(k_{m}, \mu^{(m)}\right)$.

Given $\mathfrak{s} \in \mathscr{T}_{n}^{u d}(\lambda)$, we identify $\mathfrak{s}_{i}$ with $\left(f_{i}, \mu^{(i)}\right)$ if $\mathfrak{s}_{i}=\mu^{(i)} \vdash i-2 f_{i}$. Define the partial order $\unlhd$ on $\mathscr{T}_{n}^{u d}(\lambda)$ by declaring that $\mathfrak{s} \unlhd \mathfrak{t}$ if $\mathfrak{s}_{i} \unlhd \mathfrak{t}_{i}$ for all $1 \leq i \leq n$. Write $\mathfrak{s} \triangleleft \mathfrak{t}$ if $\mathfrak{s} \unlhd \mathfrak{t}$ and $\mathfrak{s} \neq \mathfrak{t}$.

Define

$$
\begin{aligned}
& N^{\unrhd \mu^{(i)}}=R-\operatorname{span}\left\{\mathfrak{m}_{\mathfrak{t}}\right.\left.\left(\bmod \mathscr{B}_{n}(\delta)^{\triangleright(f, \lambda)}\right) \mid \mathfrak{t} \in \mathscr{T}_{n}^{u d}(\lambda), \mathfrak{t}_{n-1} \unrhd \mu^{(i)}\right\}, \\
& N^{\triangleright \mu^{(i)}}=R-\operatorname{span}\left\{\mathfrak{m}_{\mathfrak{t}} \quad\left(\bmod \mathscr{B}_{n}(\delta)^{\triangleright(f, \lambda)}\right) \mid \mathfrak{t} \in \mathscr{T}_{n}^{u d}(\lambda), \mathfrak{t}_{n-1} \triangleright \mu^{(i)}\right\} .
\end{aligned}
$$

Under the previous identification, that $\mathfrak{t}_{n-1} \unrhd \mu^{(i)}$ is equivalent to either $\left|\mathfrak{t}_{n-1}\right|<\left|\mu^{(i)}\right|$ or $\left|\mathfrak{t}_{n-1}\right|=\left|\mu^{(i)}\right|$ and $\mathfrak{t}_{n-1} \unrhd \mu^{(i)}$ under the usual dominance order.

It is well known that $\mathscr{B}_{n}(\delta)$ can be defined via Brauer diagrams. In [MW00, Morton and Wassermann proved that a Brauer algebra defined by Brauer diagrams is isomorphic to $\mathscr{B}_{n}(\delta)$ defined in section 2 . Therefore, the subalgebra of $\mathscr{B}_{n}(\delta)$ generated by $s_{i}, e_{i}, 1 \leq i \leq n-2$, is isomorphic to $\mathscr{B}_{n-1}(\delta)$.

Theorem 2.5. Eny05 Let $\mathscr{B}_{n}(\delta)$ be a Brauer algebra over a commutative ring $R$. Assume that $(f, \lambda) \in \Lambda_{n}$.

a) $\left\{\mathfrak{m}_{\mathfrak{t}}\left(\bmod \mathscr{B}_{n}(\delta)^{\triangleright(f, \lambda)}\right) \mid \mathfrak{t} \in \mathscr{T}_{n}^{u d}(\lambda)\right\}$ is an R-basis of $\Delta(f, \lambda)$.

b) Both $N^{\unrhd \mu^{(i)}}$ and $N^{\triangleright \mu^{(i)}}$ are $\mathscr{B}_{n-1}(\delta)$-submodules of $\Delta(f, \lambda)$.

c) The R-linear map $\phi: N^{\unrhd \mu^{(i)}} / N^{\triangleright \mu^{(i)}} \rightarrow \Delta\left(k_{i}, \mu^{(i)}\right)$ sending $\mathfrak{m}_{\mathfrak{t}}$ $\left(\bmod N^{\triangleright \mu^{(i)}}\right)$ to $\mathfrak{m}_{\mathfrak{t}_{n-1}}\left(\bmod \mathscr{B}_{n-1}(\delta)^{\triangleright\left(k_{i}, \mu^{(i)}\right)}\right)$ is an isomorphism of $\mathscr{B}_{n-1}(\delta)$-modules.

Definition 2.6. Given $\mathfrak{s}, \mathfrak{t} \in \mathscr{T}_{n}^{u d}(\lambda)$, define $\mathfrak{m}_{\mathfrak{s , t}}=\sigma\left(b_{\mathfrak{s}}\right) \mathfrak{m}_{\lambda} b_{\mathfrak{t}}$, where $\sigma: \mathscr{B}_{n}(\delta) \rightarrow \mathscr{B}_{n}(\delta)$ is the $R$-linear anti-involution on $\mathscr{B}_{n}(\delta)$ given in Theorem 2.2 .

Theorem 2.7. Let $\mathscr{B}_{n}(\delta)$ be a Brauer algebra over a commutative ring $R$. Then

a) $\mathscr{M}_{n}=\left\{\mathfrak{m}_{\mathfrak{s , t} \mathfrak{t}} \mid \mathfrak{s}, \mathfrak{t} \in \mathscr{T}_{n}^{u d}(\lambda), \lambda \vdash n-2 f, 0 \leq f \leq\left\lfloor\frac{n}{2}\right\rfloor\right\}$ is a free $R$-basis of $\mathscr{B}_{n}(\delta)$.

b) $\sigma\left(\mathfrak{m}_{\mathfrak{s}, \mathfrak{t}}\right)=\mathfrak{m}_{\mathfrak{t}, \mathfrak{s}}$ for all $\mathfrak{s}, \mathfrak{t} \in \mathscr{T}_{n}^{u d}(\lambda)$ and all $(f, \lambda) \in \Lambda_{n}$.

c) Let $\widetilde{\mathscr{B}_{n}(\delta)}{ }^{(f, \lambda)}$ be the free $R$-submodule of $\mathscr{B}_{n}(\delta)$ generated by $\mathfrak{m}_{\tilde{\mathfrak{s}}, \tilde{\mathfrak{t}}}$ with $\tilde{\mathfrak{s}}, \tilde{\mathfrak{t}} \in \mathscr{T}_{n}^{\text {ud }}(\mu)$ and $\left(\frac{n-|\mu|}{2}, \mu\right) \triangleright(f, \lambda)$. Then $\widetilde{\mathscr{B}_{n}(\delta)}{ }^{\triangleright(f, \lambda)}=$ $\mathscr{B}_{n}(\delta)^{\triangleright(f, \lambda)}$. 
d) For all $\mathfrak{s}, \mathfrak{t} \in \mathscr{T}_{n}^{\text {ud }}(\lambda)$ with $(f, \lambda) \in \Lambda_{n}$, and all $h \in \mathscr{B}_{n}(\delta)$, there exist scalars $a_{\mathfrak{u}} \in R$ which are independent of $\mathfrak{s}$, such that

$$
\mathfrak{m}_{\mathfrak{s}, \mathfrak{t}} h \equiv \sum_{\mathfrak{u}} a_{\mathfrak{u}} \mathfrak{m}_{\mathfrak{s}, \mathfrak{u}} \quad\left(\bmod \mathscr{B}_{n}(\delta)^{\triangleright(f, \lambda)}\right)
$$

Proof. If $(f, \lambda) \in \Lambda_{n}$ is maximal, then (c) holds since both $\mathscr{B}_{n}(\delta)^{\triangleright(f, \lambda)}$ and $\widetilde{\mathscr{B}_{n}(\delta)}{ }^{\triangleright(f, \lambda)}$ are equal to zero. In general, take a minimal element $(k, \mu) \in \Lambda_{n}$

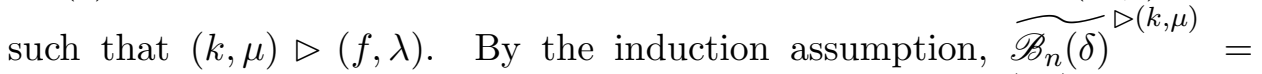
$\mathscr{B}_{n}(\delta)^{\triangleright(k, \mu)}$. By Theorem 2.5)(a) and $h \mathfrak{m}_{\mu}=\sigma\left(\mathfrak{m}_{\mu} \sigma(h)\right), C_{(\mathbf{s}, u)(\mathbf{t}, v)}^{(k, \mu)}$ can be expressed as a linear combination of $\mathfrak{m}_{\mathfrak{s t}}$ with $\mathfrak{s}, \mathfrak{t} \in \mathscr{T}_{n}^{u d}(\mu)$ module the

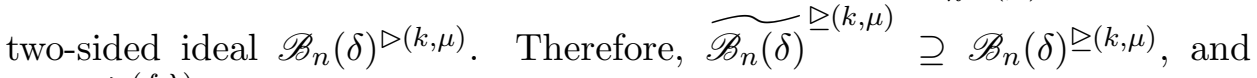

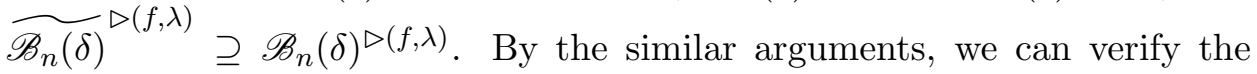
inverse inclusion. This proves (c). (d) follows from Theorem 2.5(a).

Let $N$ be the $R$-module generated by $\mathscr{M}_{n}$. By (c) and Theorem 2.5)(a), $\mathfrak{m}_{\mathfrak{s t}} h=\sigma\left(b_{\mathfrak{s}}\right)\left(\mathfrak{m}_{\lambda} b_{\mathfrak{t}} h\right) \in N$ for any $h \in \mathscr{B}_{n}(\delta)$. Therefore, $N$ is a right $\mathscr{B}_{n}(\delta)$ module. Since $1=x_{\lambda} \in N$ for $\lambda=(1,1, \cdots, 1) \vdash n, N=\mathscr{B}_{n}(\delta)$. Notice that $\# \mathscr{M}_{n}=\sum_{(f, \lambda) \in \Lambda_{n}} \# \mathscr{T}_{n}^{u d}(\lambda)^{2}=\operatorname{rank} \mathscr{B}_{n}(\delta), \mathscr{M}_{n}$ has to be an $R$-basis of $\mathscr{B}_{n}(\delta)$. Finally, (b) follows from the equality $\sigma\left(\mathfrak{m}_{\lambda}\right)=\mathfrak{m}_{\lambda}$.

We call $\mathscr{M}_{n}$ the Jucys-Murphy basis of $\mathscr{B}_{n}(\delta)$. It is a cellular basis of $\mathscr{B}_{n}(\delta)$ over $R$. In order to simplify the notation, we use $\mathfrak{m}_{\mathfrak{t}}$ instead of $\mathfrak{m}_{\mathfrak{t}}$ $\left(\bmod \mathscr{B}_{n}(\delta)^{\triangleright(f, \lambda)}\right)$ if there is no confusion.

In GL96, Graham and Lehrer proved that there is a symmetric invariant bilinear form $\langle, \quad\rangle: \Delta(f, \lambda) \times \Delta(f, \lambda) \rightarrow R$. In our case, we use $\mathscr{M}_{n}$ to define such a bilinear form on $\Delta(f, \lambda)$. More explicitly, $\left\langle\mathfrak{m}_{\mathfrak{s}}, \mathfrak{m}_{\mathfrak{t}}\right\rangle \in R$ is determined by

$$
\mathfrak{m}_{\tilde{\mathfrak{s}} \mathfrak{s}} \mathfrak{m}_{\tilde{\mathfrak{t t}}} \equiv\left\langle\mathfrak{m}_{\mathfrak{s}}, \mathfrak{m}_{\mathfrak{t}}\right\rangle \mathfrak{m}_{\tilde{\mathfrak{s}} \tilde{\mathfrak{t}}}\left(\bmod \mathscr{B}_{n}(\delta)^{\triangleright(f, \lambda)}\right), \quad \text { for some } \tilde{\mathfrak{s}}, \tilde{\mathfrak{t}} \in \mathscr{T}_{n}^{u d}(\lambda)
$$

By Theorem 2.7(d), the above symmetric invariant bilinear form is independent of $\tilde{\mathfrak{s}}, \tilde{\mathfrak{t}} \in \mathscr{T}_{n}^{u d}(\lambda)$. The Gram matrix $G_{f, \lambda}$ associated to $\Delta(f, \lambda)$ is the $k \times k$ matrix with $k=\operatorname{rank} \Delta(f, \lambda)$ such that the $(\mathfrak{t}, \mathfrak{s})$-entry is $\left\langle\mathfrak{m}_{\mathfrak{t}}, \mathfrak{m}_{\mathfrak{s}}\right\rangle$.

For any partition $\lambda=\left(\lambda_{1}, \lambda_{2}, \cdots,\right)$, let $\lambda^{\prime}=\left(\lambda_{1}^{\prime}, \lambda_{2}^{\prime}, \cdots,\right)$ be its dual partition. Then

$$
\operatorname{rank} \Delta(f, \lambda)=\frac{n !(2 f-1) ! !}{(2 f) ! \prod_{(i, j) \in \lambda} h_{i, j}^{\lambda}},
$$

where $h_{i, j}^{\lambda}=\lambda_{i}+\lambda_{j}^{\prime}-i-j+1$. In general, $\operatorname{rank} \Delta(f, \lambda)$ is a very large integer. Therefore, it is very difficult to compute the Gram matrix $G_{f, \lambda}$ directly.

In GL96, Graham and Lehrer proved that a cellular algebra is (split) semisimple if and only if the Gram determinant associated to each cell module is not equal to zero. Via it, we proved the following theorem. 
Theorem 2.8. Rui05, RS06a Let $\mathscr{B}_{n}(\delta)$ be a Brauer algebra over an arbitrary field $F$. Define $e=+\infty$ (resp. $p$ ) if the characteristic of $F$ is zero (resp. p>0).

a) Suppose $\delta \neq 0$. Then $\mathscr{B}_{n}(\delta)$ is semisimple if and only if $\delta \notin\{i,-2 i \mid 1 \leq i \leq n-2, i \in \mathbb{Z}\} \cup\{j \in \mathbb{Z} \mid 4-n \leq j \leq-1\}$ and $e \nmid n !$.

b) $\mathscr{B}_{n}(0)$ is semisimple if and only if $n \in\{1,3,5\}$ and $e \nmid n$ !.

We remark that Theorem 2.8 has been generalised to cyclotomic Brauer algebras in RY04, RX05].

For any $\mathfrak{t} \in \mathscr{T}_{n}^{u d}(\lambda)$ with $(f, \lambda) \in \Lambda_{n}$, define the residue of $k$ in $\mathfrak{t}$ to be the scalar $c_{\mathfrak{t}}(k) \in R$ such that

$$
c_{\mathfrak{t}}(k)= \begin{cases}\frac{\delta-1}{2}+j-i, & \text { if } \mathfrak{t}_{k}=\mathfrak{t}_{k-1} \cup\{(i, j)\}, \\ -\frac{\delta-1}{2}+i-j, & \text { if } \mathfrak{t}_{k-1}=\mathfrak{t}_{k} \cup\{(i, j)\} .\end{cases}
$$

We also define

$$
c_{\lambda}(p)= \begin{cases}\frac{\delta-1}{2}+j-i, & \text { if } p=(i, j) \text { is an addable node of } \lambda, \\ -\frac{\delta-1}{2}-j+i, & \text { if } p=(i, j) \text { is a removable node of } \lambda .\end{cases}
$$

In [Naz96], Nazarov defined the Jucys-Murphy elements $x_{i}$ of $\mathscr{B}_{n}(\delta)$ by declaring that

$$
x_{i}= \begin{cases}\frac{\delta-1}{2}, & \text { if } i=1, \\ \frac{\delta-1}{2}+\sum_{k=1}^{i-1}\left(s_{k, i} s_{i-1, k}-s_{k, i-2} s_{i-1, k} e_{i-1} s_{k, i-1} s_{i-2, k}\right), & \text { if } 2 \leq i \leq n .\end{cases}
$$

Remark 2.9. In the definitions of $c_{\mathfrak{t}}(k)$ and $x_{k}$, we assume $\frac{1}{2} \in R$. In fact, we do not need this assumption since $x_{k}$ can be defined by using 0 instead of $\frac{\delta-1}{2}$. In this situation, we have to define $c_{\mathfrak{t}}(k)=j-i$ if $\mathfrak{t}_{k}=\mathfrak{t}_{k-1} \cup\{(i, j)\}$ and $c_{\mathfrak{t}}(k)=1-\delta-j+i$ if $\mathfrak{t}_{k-1}=\mathfrak{t}_{k} \cup\{(i, j)\}$.

Nazarov proved the following Lemma for $\mathscr{B}_{n}(\delta)$ over $\mathbb{C}$. It holds in general AMR06.

Lemma 2.10. Naz96, §4]
a) $e_{i} x_{j}=x_{j} e_{i}, j \neq i, i+1$,
e) $e_{i}\left(x_{i}+x_{i+1}\right)=0,1 \leq i<n$,
b) $s_{i} x_{j}=x_{j} s_{i}, j \neq i, i+1$,
f) $\left(x_{i}+x_{i+1}\right) e_{i}=0,1 \leq i<n$,
c) $s_{i} x_{i}-x_{i+1} s_{i}=e_{i}-1,1 \leq i<n$,
g) $x_{i} x_{j}=x_{j} x_{i}, 1 \leq i, j \leq n$.
d) $x_{i} s_{i}-s_{i} x_{i+1}=e_{i}-1,1 \leq i<n$,

Theorem 2.11. Eny05, 10.7] Given $\mathfrak{s}, \mathfrak{t} \in \mathscr{T}_{n}^{u d}(\lambda)$, with $(f, \lambda) \in \Lambda_{n}$, let $\mathfrak{m}_{\mathfrak{s t}} \in \mathscr{B}_{n}(\delta)$ be defined in Definition [2.6. Then

$$
\mathfrak{m}_{\mathfrak{s t}} x_{k} \equiv c_{\mathfrak{t}}(k) \mathfrak{m}_{\mathfrak{s t}}+\sum_{\substack{\mathfrak{u} \in \mathscr{T}_{n}^{u d}(\lambda) \\ \mathfrak{u} \triangleright \mathfrak{t}}} a_{\mathfrak{u}} \mathfrak{m}_{\mathfrak{s u}} \quad\left(\bmod \mathscr{B}_{n}(\delta)^{\triangleright(f, \lambda)}\right) .
$$

Theorem 2.11 was proved in Eny05, 10.7] under the assumption $\mathfrak{s}=\mathfrak{t}^{\lambda}$. In general, it follows from this special case since multiplying an element on the left side of $\mathfrak{m}_{\mathfrak{t}}\left(\bmod \mathscr{B}_{n}(\delta)^{\triangleright(f, \lambda)}\right)$ gives a homomorphism of right $\mathscr{B}_{n}(\delta)$-module. 


\section{Orthogonal Representations for $\mathscr{B}_{n}(\delta)$}

We always assume that $F$ is a field which satisfies the assumptions 3.1 in this section. The main purpose of this section is to construct an orthogonal basis. We remark that many results in this section are motivated by [Mat04.

Assumption 3.1. Suppose that $F$ is a field of characteristic $p$ where either $p=0$ or $p>2 n$. Suppose $\delta \in F$ such that $|c| \geq 2 n+1$ whenever $\delta-c \cdot 1_{F}=0$ for some $c \in \mathbb{Z}$.

For example, $\mathbb{C}(\delta)$ satisfies assumption 3.1, where $\delta$ is an indeterminate.

Definition 3.2. Suppose $1 \leq k \leq n-1$ and $(f, \lambda) \in \Lambda_{n}$. Define an equivalence relation $\stackrel{k}{\sim}$ on $\mathscr{T}_{n}^{u d}(\lambda)$ by declaring that $\mathfrak{t} \stackrel{k}{\sim} \mathfrak{s}$ if $\mathfrak{t}_{j}=\mathfrak{s}_{j}$ whenever $1 \leq j \leq n$ and $j \neq k$, for $\mathfrak{s}, \mathfrak{t} \in \mathscr{T}_{n}^{u d}(\lambda)$.

The following result is a special case of [AMR06, 4.2].

Lemma 3.3. Suppose $\mathfrak{s} \in \mathscr{T}_{n}^{u d}(\lambda)$ with $\mathfrak{s}_{k-1}=\mathfrak{s}_{k+1}$. Then there is a bijection between the set of all addable and removable nodes of $\mathfrak{s}_{k+1}$ and the set $\left\{\mathfrak{t} \in \mathscr{T}_{n}^{u d}(\lambda) \mid \mathfrak{t} \stackrel{k}{\sim} \mathfrak{s}\right\}$.

Suppose $\lambda$ and $\mu$ are partitions. We write $\lambda \ominus \mu=\alpha$ if either $\lambda \supset \mu$ and $\lambda \backslash \mu=\alpha$ or $\lambda \subset \mu$ and $\mu \backslash \lambda=\alpha$.

Lemma 3.4. Suppose that $F$ is a field which satisfies the assumption 3.1. Assume that $\mathfrak{s}, \mathfrak{t} \in \mathscr{T}_{n}^{u d}(\lambda)$ with $(f, \lambda) \in \Lambda_{n}$.

a) $\mathfrak{s}=\mathfrak{t}$ if and only if $c_{\mathfrak{s}}(k)=c_{\mathfrak{t}}(k)$ for $1 \leq k \leq n$.

b) If $\mathfrak{t}_{k-1} \neq \mathfrak{t}_{k+1}$, then $c_{\mathfrak{t}}(k) \pm c_{\mathfrak{t}}(k+1) \neq 0$.

c) If $\mathfrak{t}_{k-1}=\mathfrak{t}_{k+1}$, then $c_{\mathfrak{t}}(k) \pm c_{\mathfrak{s}}(k) \neq 0$ whenever $\mathfrak{s} \stackrel{k}{\sim} \mathfrak{t}$ and $\mathfrak{s} \neq \mathfrak{t}$.

d) If $\mathfrak{t}_{k-1}=\mathfrak{t}_{k+1}$, then $2 c_{\mathfrak{t}}(k)+1 \neq 0$.

Proof. The "only if " part of (a) is trivial. We prove the "if" part of (a) as follows. (b)-(d) can be proved similarly. We leave the details to the reader.

By the definition of the $n$-updown tableau, $\mathfrak{t}_{1}=(1)$ for any $\mathfrak{t} \in \mathscr{T}_{n}^{u d}(\lambda)$. Assume $\mathfrak{t}_{k-1}=\mathfrak{s}_{k-1}$ and $\mathfrak{t}_{k} \ominus \mathfrak{t}_{k-1}=(i, j)$ and $\mathfrak{s}_{k} \ominus \mathfrak{s}_{k-1}=\left(i^{\prime}, j^{\prime}\right)$.

If the sign of $\delta$ in $c_{\mathfrak{s}}(k)$ and $c_{\mathfrak{t}}(k)$ are different, then, $n \geq 3$ and $(i, j) \neq$ $\left(i^{\prime}, j^{\prime}\right)$ since one node of a Young diagram can not be an addable and a removable node simultaneously. We write $c_{\mathfrak{t}}(k)=\frac{\delta-1}{2}+j-i$ and $c_{\mathfrak{s}}(k)=$ $-\frac{\delta-1}{2}+i^{\prime}-j^{\prime}$ without loss of generality. In this situation, $\lambda \vdash n-2 f$ and $f>0$. The maximal (resp. minimal) value of $(i-j)+\left(i^{\prime}-j^{\prime}\right)$ is $2 n-5$ (resp. $5-2 n$ ). In the first case, $\lambda=(1,1, \cdots, 1) \vdash n-2$ and $\left\{(i, j),\left(i^{\prime}, j^{\prime}\right)\right\}=\{(n-1,1),(n-2,1)\}$. In the second case, $\lambda=(n-2) \vdash n-2$ and $\left\{(i, j),\left(i^{\prime}, j^{\prime}\right)\right\}=\{(1, n-1),(1, n-2)\}$. In any case, $\delta=1+i^{\prime}-j^{\prime}+i-j$ if $c_{\mathfrak{s}}(k)=c_{\mathfrak{t}}(k)$. We have $\left|1+\left(i^{\prime}-j^{\prime}+i-j\right)\right| \leq 2 n-4$, which contradicts the assumption 3.1 . 
Suppose that the sign of $\delta$ in $c_{\mathfrak{s}}(k)$ and $c_{\mathfrak{t}}(k)$ are the same. Then both $(i, j)$ and $\left(i^{\prime}, j^{\prime}\right)$ are either addable nodes or removable nodes of $\mathfrak{t}_{k-1}=\mathfrak{s}_{k-1}$. If $\mathfrak{s}_{k} \neq \mathfrak{t}_{k}$, then $(i, j) \neq\left(i^{\prime}, j^{\prime}\right)$. In this situation, such two nodes can not be in the same diagonal of the partition which is obtained from $\mathfrak{t}_{k-1}$ by adding (if $\mathfrak{t}_{k} \supset \mathfrak{t}_{k-1}$ ) or removing (if $\left.\mathfrak{t}_{k} \subset \mathfrak{t}_{k-1}\right)(i, j)$ and $\left(i^{\prime}, j^{\prime}\right)$. Therefore, $i-j \neq i^{\prime}-j^{\prime}$ and $c_{\mathfrak{s}}(k) \neq c_{\mathfrak{t}}(k)$, a contradiction.

Following [Mur83], we make the following definition.

Definition 3.5. Let $\mathscr{B}_{n}(\delta)$ be a Brauer algebra over a field $F$. Define

a) $\mathscr{R}(k)=\left\{c_{\mathfrak{t}}(k) \mid \mathfrak{t} \in \mathscr{T}_{n}^{u d}(\lambda),(f, \lambda) \in \Lambda_{n}\right\}$ for any $k \in \mathbb{Z}, 1 \leq k \leq n$.

b) $F_{\mathfrak{t}}=\prod_{k=1}^{n} \prod_{r \in \mathscr{R}(k)} \frac{x_{k}-r}{c_{\mathfrak{t}}(k)-r}$

c) $f_{\mathfrak{s t}}=F_{\mathfrak{s}} \mathfrak{m}_{\mathfrak{s t}} F_{\mathfrak{t}}$,

d) $f_{\mathfrak{s}}=\mathfrak{m}_{\mathfrak{s}} F_{\mathfrak{s}}$,

where $\mathfrak{s}, \mathfrak{t} \in \mathscr{T}_{n}^{u d}(\lambda)$ and $(f, \lambda) \in \Lambda_{n}$.

In (d), we use $\mathfrak{m}_{\mathfrak{s}}$ instead of $\mathfrak{m}_{\mathfrak{s}}\left(\bmod \mathscr{B}_{n}(\delta)^{\triangleright(f, \lambda)}\right)$. Therefore, (d) should be read as $f_{\mathfrak{s}} \equiv \mathfrak{m}_{\mathfrak{s}} F_{\mathfrak{s}}\left(\bmod \mathscr{B}_{n}(\delta)^{\triangleright(f, \lambda)}\right)$. In what follows, we will omit $\left(\bmod \mathscr{B}_{n}(\delta)^{\triangleright(f, \lambda)}\right)$ for the simplification in exposition and notation.

Lemma 3.6. Let $\mathscr{B}_{n}(\delta)$ be a Brauer algebra over a field $F$. Suppose that $\mathfrak{t} \in \mathscr{T}_{n}^{u d}(\lambda)$ with $(f, \lambda) \in \Lambda_{n}$.

a) $f_{\mathfrak{t}}=\mathfrak{m}_{\mathfrak{t}}+\sum_{\mathfrak{s} \in \mathscr{T}_{n}^{u d}(\lambda)} a_{\mathfrak{s}} \mathfrak{m}_{\mathfrak{s}}$, and $\mathfrak{s} \triangleright \mathfrak{t}$ if $a_{\mathfrak{s}} \neq 0$.

b) $\mathfrak{m}_{\mathfrak{t}}=f_{\mathfrak{t}}+\sum_{\mathfrak{s} \in \mathscr{T}_{n}^{u d}(\lambda)} b_{\mathfrak{s}} f_{\mathfrak{s}}$, and $\mathfrak{s} \triangleright \mathfrak{t}$ if $b_{\mathfrak{s}} \neq 0$.

c) $f_{\mathfrak{t}} x_{k}=c_{\mathfrak{t}}(k) f_{\mathfrak{t}}$, for any integer $k, 1 \leq k \leq n$.

d) $f_{\mathfrak{t}} F_{\mathfrak{s}}=\delta_{\mathfrak{s t}} f_{\mathfrak{t}}$ for all $\mathfrak{s} \in \mathscr{T}_{n}^{u d}(\mu)$ with $\left(\frac{n-|\mu|}{2}, \mu\right) \in \Lambda_{n}$.

Proof. (a) follows from Theorem 2.11. Since the transition matrix from $\left\{f_{\mathfrak{s}} \mid \mathfrak{s} \in \mathscr{T}_{n}^{u d}(\lambda)\right\}$ to $\left\{\mathfrak{m}_{\mathfrak{s}} \mid \mathfrak{s} \in \mathscr{T}_{n}^{u d}(\lambda)\right\}$ is upper uni-triangular, so is its inverse. This prove (b). (c) can be proved by the arguments in [Mat99, 3.35]. The only difference is that we need use $\# \mathscr{T}_{n}^{u d}(\lambda)$ instead of $\# \mathscr{T}_{n}^{\text {std }}(\lambda)$ in the proof of [Mat99, 3.35]. (d) follows from (c).

By Lemma 3.6)(a), $\left\{f_{\mathfrak{t}} \mid \mathfrak{t} \in \mathscr{T}_{n}^{u d}(\lambda)\right\}$ is an $F$-basis for $\Delta(f, \lambda)$. Such a basis will be called an orthogonal basis of $\Delta(f, \lambda)$.

The following two results follow from Lemma 3.6.

Corollary 3.7. Let $\mathscr{B}_{n}(\delta)$ be a Brauer algebra over a field $F$. Let $G_{f, \lambda}$ (resp. $\tilde{G}_{f, \lambda}$ ) be the Gram matrix associated to the cell module $\Delta(f, \lambda)$ with $(f, \lambda) \in \Lambda_{n}$, which is defined via its Jucys-Murphy (resp. orthogonal) basis. Then $\operatorname{det} G_{f, \lambda}=\operatorname{det} \tilde{G}_{f, \lambda}$.

Corollary 3.8. Let $\mathscr{B}_{n}(\delta)$ be a Brauer algebra over a field F. Keep the setup in Theorem 2.5.

a) $\left\{f_{\mathfrak{t}} \mid \mathfrak{t} \in \mathscr{T}_{n}^{u d}(\lambda), \mathfrak{t}_{n-1} \unrhd \mu^{(i)}\right\}$ is an $F$-basis of $N^{\unrhd \mu^{(i)}}$. 
b) $\left\{f_{\mathfrak{t}} \mid \mathfrak{t} \in \mathscr{T}_{n}^{u d}(\lambda), \mathfrak{t}_{n-1} \triangleright \mu^{(i)}\right\}$ is an $F$-basis of $N^{\triangleright \mu^{(i)}}$.

c) The F-linear map $\phi: N^{\unrhd \mu^{(i)}} / N^{\triangleright \mu^{(i)}} \rightarrow \Delta\left(k_{i}, \mu^{(i)}\right)$ sending $f_{\mathfrak{t}}+N^{\triangleright \mu^{(i)}}$ to $f_{\mathfrak{t}_{n-1}}$ is an isomorphism of $\mathscr{B}_{n-1}(\delta)$-modules.

The following result can be proved by using the arguments in [Mat04.

Lemma 3.9. Let $\mathscr{B}_{n}(\delta)$ be a Brauer algebra over a field $F$.

a) $\left\{f_{\mathfrak{s t}} \mid \mathfrak{s}, \mathfrak{t} \in \mathscr{T}_{n}^{\text {ud }}(\lambda),(f, \lambda) \in \Lambda_{n}\right\}$ is a basis of $\mathscr{B}_{n}(\delta)$ over $F$.

b) $f_{\mathfrak{s t}} x_{k}=c_{\mathfrak{t}}(k) f_{\mathfrak{s t}}$ for any $\mathfrak{s}, \mathfrak{t} \in \mathscr{T}_{n}^{u d}(\lambda)$ with $\left(\frac{n-|\lambda|}{2}, \lambda\right) \in \Lambda_{n}$.

c) $f_{\mathfrak{s t}} F_{\mathfrak{u}}=\delta_{\mathfrak{t u}} f_{\mathfrak{s t}}$ for any $\mathfrak{s}, \mathfrak{t} \in \mathscr{T}_{n}^{\text {ud }}(\lambda)$ with $\left(\frac{n-|\lambda|}{2}, \lambda\right) \in \Lambda_{n}$ and any $\mathfrak{u} \in \mathscr{T}_{n}^{u d}(\mu)$ with $\left(\frac{n-|\mu|}{2}, \mu\right) \in \Lambda_{n}$.

d) Suppose $\mathfrak{s}, \mathfrak{t} \in \mathscr{T}_{n}^{u d}(\lambda)$ with $\left(\frac{n-|\lambda|}{2}, \lambda\right) \in \Lambda_{n}$ and $\mathfrak{u}, \mathfrak{v} \in \mathscr{T}_{n}^{u d}(\mu)$ with $\left(\frac{n-|\mu|}{2}, \mu\right) \in \Lambda_{n}$. Then $f_{\mathfrak{s t}} f_{\mathfrak{u v}}=\delta_{\mathfrak{t u}}\left\langle f_{\mathfrak{t}}, f_{\mathfrak{t}}\right\rangle f_{\mathfrak{s v}}$ for some scalar $\left\langle f_{\mathfrak{t}}, f_{\mathfrak{t}}\right\rangle \in$ F.

Under the assumption 3.1, $\mathscr{B}_{n}(\delta)$ is semisimple over $F$ (see Theorem 2.8). Therefore, $\operatorname{det} G_{f, \lambda} \neq 0$ for all $(f, \lambda) \in \Lambda_{n}$. By Corollary 3.7,

$$
\left\langle f_{\mathfrak{t}}, f_{\mathfrak{t}}\right\rangle \neq 0, \text { for all } \mathfrak{t} \in \mathscr{T}_{n}^{u d}(\lambda) .
$$

Lemma 3.11. Let $\mathscr{B}_{n}(\delta)$ be a Brauer algebra over a field $F$. Suppose $\mathfrak{t} \in$ $\mathscr{T}_{n}^{u d}(\lambda)$ and $1 \leq k \leq n-1$.

a) Let $f_{\mathfrak{t}} s_{k}=\sum_{\mathfrak{s} \in \mathscr{T}_{n}^{u d}(\lambda)} s_{\mathfrak{t s}}(k) f_{\mathfrak{s}}$. If $s_{\mathfrak{t s}}(k) \neq 0$, then $\mathfrak{s} \stackrel{k}{\sim} \mathfrak{t}$.

b) Let $f_{\mathfrak{t}} e_{k}=\sum_{\mathfrak{s} \in \mathscr{T}_{n}^{u d}(\lambda)} e_{\mathfrak{t s}}(k) f_{\mathfrak{s}}$. If $e_{\mathfrak{t s}}(k) \neq 0$, then $\mathfrak{s} \stackrel{k}{\sim} \mathfrak{t}$.

Proof. By Lemma 2.10(a)-(b), $c_{\mathfrak{s}}(j)=c_{\mathfrak{t}}(j)$ for $j \neq k, k+1$ if either $s_{\mathfrak{t s}}(k) \neq$ 0 or $e_{\mathfrak{t} \mathfrak{s}}(k) \neq 0$. Notice that $\mathfrak{s}_{0}=\mathfrak{t}_{0}=\varnothing$ and $\mathfrak{s}_{n}=\mathfrak{t}_{n}=\lambda$. Applying Lemma 3.4(a) to the sequences $\left(\mathfrak{s}_{0}, \mathfrak{s}_{1}, \cdots, \mathfrak{s}_{k-1}\right)$ and $\left(\mathfrak{s}_{k+1}, \cdots, \mathfrak{s}_{n}\right)$, we have $\mathfrak{s} \stackrel{k}{\sim} \mathfrak{t}$, as required.

Lemma 3.12. Let $\mathscr{B}_{n}(\delta)$ be a Brauer algebra over a field $F$. Suppose $\mathfrak{t} \in$ $\mathscr{T}_{n}^{u d}(\lambda)$. If $\mathfrak{t}_{k-1} \neq \mathfrak{t}_{k+1}$, then $f_{\mathfrak{t}} e_{k}=0$.

Proof. By Lemma 2.10(f), $f_{\mathfrak{t}}\left(x_{k}+x_{k+1}\right) e_{k}=\left(c_{\mathfrak{t}}(k)+c_{\mathfrak{t}}(k+1)\right) f_{\mathfrak{t}} e_{k}=0$. If $f_{\mathfrak{t}} e_{k} \neq 0$, then $c_{\mathfrak{t}}(k)+c_{\mathfrak{t}}(k+1)=0$, which contradicts Lemma 3.4 (b).

The following result can be proved easily.

Lemma 3.13. Suppose $\mathfrak{t} \in \mathscr{T}_{n}^{u d}(\lambda)$ with $\mathfrak{t}_{k-1} \neq \mathfrak{t}_{k+1}$.

a) If $\mathfrak{t}_{k} \ominus \mathfrak{t}_{k-1}$ and $\mathfrak{t}_{k+1} \ominus \mathfrak{t}_{k}$ are neither in the same row nor in the same column, then there is a unique up-down tableau in $\mathscr{T}_{n}^{u d}(\lambda)$, denoted by $\mathfrak{t}_{k}$, such that $\mathfrak{t}_{k} \stackrel{k}{\sim} \mathfrak{t}$ and $c_{\mathfrak{t}}(k)=c_{\mathfrak{t s}_{k}}(k+1)$ and $c_{\mathfrak{t}}(k+1)=c_{\mathfrak{t s}_{k}}(k)$.

b) If $\mathfrak{t}_{k} \ominus \mathfrak{t}_{k-1}$ and $\mathfrak{t}_{k+1} \ominus \mathfrak{t}_{k}$ are either in the same row or in the same column, then $\mathfrak{s} \stackrel{k}{\sim} \mathfrak{t}$ if and only if $\mathfrak{s}=\mathfrak{t}$. 
Lemma 3.14. Let $\mathscr{B}_{n}(\delta)$ be a Brauer algebra over a field $F$. Suppose $\mathfrak{t} \in$ $\mathscr{T}_{n}^{u d}(\lambda)$ with $\mathfrak{t}_{k-1} \neq \mathfrak{t}_{k+1}$. If $\mathfrak{t}_{k}$ exists, then

$$
f_{\mathfrak{t}} s_{k}=\frac{1}{c_{\mathfrak{t}}(k+1)-c_{\mathfrak{t}}(k)} f_{\mathfrak{t}}+s_{\mathfrak{t}, \mathfrak{t}_{k}}(k) f_{\mathfrak{t s}_{k}},
$$

where

$$
s_{\mathfrak{t}, \mathfrak{t s}_{k}}(k)= \begin{cases}1, & \text { if } \mathfrak{t} s_{k} \triangleleft \mathfrak{t}, \\ 1-\frac{1}{\left(c_{\mathfrak{t}}(k+1)-c_{\mathfrak{t}}(k)\right)^{2}}, & \text { if } \mathfrak{t} s_{k} \triangleright \mathfrak{t} .\end{cases}
$$

Proof. Write $f_{\mathfrak{t}} s_{k}=\sum_{\mathfrak{s} \in \mathscr{T}_{n}^{u d}(\lambda)} s_{\mathfrak{t s}}(k) f_{\mathfrak{s}}$. Since $s_{k} x_{k}=x_{k+1} s_{k}+e_{k}-1$ and $f_{\mathfrak{t}} e_{k}=0$ (see Lemma 3.12),$f_{\mathfrak{t}} s_{k} x_{k}=c_{\mathfrak{t}}(k+1) f_{\mathfrak{t}} s_{k}-f_{\mathfrak{t}}$. By Lemma 3.11, $\mathfrak{s} \stackrel{k}{\sim} \mathfrak{t}$ if $s_{\mathfrak{t} \mathfrak{s}}(k) \neq 0$. Comparing the coefficient of $f_{\mathfrak{t}}$ in $f_{\mathfrak{t}} s_{k} x_{k}$, we obtain

$$
s_{\mathfrak{t t}}(k)=\frac{1}{c_{\mathfrak{t}}(k+1)-c_{\mathfrak{t}}(k)} .
$$

The previous formula makes sense by Lemma 3.4b. Suppose $\mathfrak{s} \neq \mathfrak{t}$. Comparing the coefficient of $f_{\mathfrak{s}}$ in $f_{\mathfrak{t}} s_{k} x_{k}$, we have $s_{\mathfrak{t s}}(k) c_{\mathfrak{s}}(k)=s_{\mathfrak{t s}}(k) c_{\mathfrak{t}}(k+1)$ forcing $c_{\mathfrak{s}}(k)=c_{\mathfrak{t}}(k+1)$. If we compare the coefficient of $f_{\mathfrak{s}}$ in $f_{\mathfrak{t}} x_{k} s_{k}$, we obtain $c_{\mathfrak{s}}(k+1)=c_{\mathfrak{t}}(k)$. In other words, $\mathfrak{s}=\mathfrak{t} s_{k}$ if $s_{\mathfrak{t} \mathfrak{s}}(k) \neq 0$ with $\mathfrak{s} \neq \mathfrak{t}$. We claim that

a) $\mathfrak{m}_{\mathfrak{t}} s_{k}=\mathfrak{m}_{\mathfrak{t} s_{k}}$,

b) $f_{\mathfrak{t} s_{k}}$ does not appear in the expression of $f_{\mathfrak{u}} s_{k}$ with $\mathfrak{u} \triangleright \mathfrak{t}$.

By Lemma 3.6(a)-(b), and the above claims, we have $s_{\mathfrak{t s}}(k)=1$ for $\mathfrak{s}=$ $\mathfrak{t} s_{k} \triangleleft \mathfrak{t}$.

First, we prove (b). By Lemma 3.11(a), $f_{\mathfrak{u}} s_{k}=\sum_{\mathfrak{v} \in \mathscr{T}_{n}^{u d}(\lambda)} s_{\mathfrak{u v}}(k) f_{\mathfrak{v}}$, and $\mathfrak{v} \stackrel{k}{\sim} \mathfrak{u}$ if $s_{\mathfrak{u v}}(k) \neq 0$. If $\mathfrak{v}=\mathfrak{t} s_{k}$, then $\mathfrak{u} \stackrel{k}{\sim} \mathfrak{t}$, forcing $\mathfrak{u}_{k-1} \neq \mathfrak{u}_{k+1}$. Therefore, $\mathfrak{u} \in\left\{\mathfrak{t}, \mathfrak{t} s_{k}\right\}$ which contradicts the assumption $\mathfrak{u} \triangleright \mathfrak{t} \triangleright \mathfrak{t} s_{k}$.

Now we prove (a). Note that $\mathfrak{m}_{\mathfrak{t}} s_{k}=\mathfrak{m}_{\mathfrak{t} s_{k}}$ if and only if $\mathfrak{m}_{\mathfrak{t}}=\mathfrak{m}_{\mathfrak{t}_{k}} s_{k}$. Therefore, (a) holds although $\mathfrak{t} s_{k} \triangleright \mathfrak{t}$.

By Theorem 2.5(c), the coefficient of $\mathfrak{m}_{\mathfrak{t}_{k}}$ in $\mathfrak{m}_{\mathfrak{t}} s_{k}$ is completely determined by $\mathfrak{m}_{\mathfrak{t}_{k+1}} s_{k}$. So, we can assume $k=n-1$ without loss of generality when we prove (a).

There are four cases we have to discuss. In any case, $\mathfrak{t}_{n} \ominus \mathfrak{t}_{n-1}$ and $\mathfrak{t}_{n-1} \ominus \mathfrak{t}_{n-2}$ are neither in the same row nor in the same column of $\mathfrak{t}_{n-1}$. Otherwise, $\mathfrak{t}_{n-1}$ does not exist. Suppose $\mathfrak{t}_{n}=\lambda$ and $\mathfrak{t}_{n-1}=\mu$.

Case 1. $\mathfrak{t}_{n} \supset \mathfrak{t}_{n-1} \supset \mathfrak{t}_{n-2}$ :

There are some $k, l \in \mathbb{N}$ such that $\mathfrak{t}_{n} \backslash \mathfrak{t}_{n-1}=\left(k, \lambda_{k}\right)$ and $\mathfrak{t}_{n-1} \backslash \mathfrak{t}_{n-2}=$ $\left(l, \mu_{l}\right)$. If we write $a=2 f+\sum_{j=1}^{k} \lambda_{j}$ and $b=2 f+\sum_{j=1}^{l} \mu_{j}$, then $\mathfrak{m}_{\mathfrak{t}_{n}}=\mathfrak{m}_{\lambda} s_{a, n} s_{b, n-1} b_{\mathfrak{t}_{n-2}}$. Note that $b_{\mathfrak{t}_{n-2}} \in \mathfrak{S}_{n-2}$ (see (2.4)), $b_{\mathfrak{t}_{n-2}} s_{n-1}=$ $s_{n-1} b_{\mathfrak{t}_{n-2}}$. Therefore,

$$
\mathfrak{m}_{\mathfrak{t}} s_{n-1}=\mathfrak{m}_{\lambda} s_{a, n} s_{b, n-1} s_{n-1} b_{\mathfrak{t}_{n-2}}=\mathfrak{m}_{\lambda} s_{b, n} s_{a-1, n-1} b_{\mathfrak{t}_{n-2}}=\mathfrak{m}_{\mathfrak{t}_{n-1}}
$$

if $a>b$. The result for $a<b$ is still true since we can switch $\mathfrak{t}$ to $\mathfrak{t} s_{n-1}$ in the above argument. We remark that $a>b$ if $\mathfrak{t} s_{n-1} \triangleleft \mathfrak{t}$. 
Case 2. $\mathfrak{t}_{n} \subset \mathfrak{t}_{n-1} \supset \mathfrak{t}_{n-2}$ :

Then $\mathfrak{t}_{n-1} \backslash \mathfrak{t}_{n}=\left(k, \mu_{k}\right)$ and $\mathfrak{t}_{n-1} \backslash \mathfrak{t}_{n-2}=\left(l, \mu_{l}\right)$. Write $a_{i}=2(f-1)+$ $\sum_{j=1}^{i} \mu_{j}$. If $l>k$, then $a_{l}>a_{k}>a_{k-1}$. Therefore,

$$
\begin{aligned}
\mathfrak{m}_{\mathfrak{t}_{n}} s_{n-1} & =\mathfrak{m}_{\lambda} s_{2 f, n} \sum_{j=a_{k-1}+1}^{a_{k}} s_{2 f-1, j} s_{a_{l}, n} b_{\mathfrak{t}_{n-2}} \\
& =\mathfrak{m}_{\lambda} s_{a_{l}+1, n} s_{2 f, n-1} \sum_{j=a_{k-1}+1}^{a_{k}} s_{2 f-1, j} b_{\mathfrak{t}_{n-2}}=\mathfrak{m}_{\mathfrak{t}_{n-1}} .
\end{aligned}
$$

If $l<k$, then $a_{l} \leq a_{k-1}<a_{k}$. By braid relation,

$$
\begin{aligned}
\mathfrak{m}_{\mathfrak{t}_{n}} s_{n-1} & =\mathfrak{m}_{\lambda} s_{2 f, n} \sum_{j=a_{k-1}+1}^{a_{k}} s_{2 f-1, j} s_{a_{l}, n-1} s_{n-1} b_{\mathfrak{t}_{n-2}} \\
& =\mathfrak{m}_{\lambda} s_{2 f, n} s_{a_{l}+1, n} \sum_{j=a_{k-1}+1}^{a_{k}} s_{2 f-1, j-1} b_{\mathfrak{t}_{n-2}} \\
& =\mathfrak{m}_{\lambda} s_{a_{l}+2, n} s_{2 f, n-1} \sum_{j=a_{k-1}}^{a_{k}-1} s_{2 f-1, j} b_{\mathfrak{t}_{n-2}}=\mathfrak{m}_{\mathfrak{t}_{s_{n-1}}} .
\end{aligned}
$$

Finally, we remark that $\mathfrak{t}_{n-1} \triangleleft \mathfrak{t}$ is not compatible with $\mathfrak{t}_{n} \subset \mathfrak{t}_{n-1} \supset \mathfrak{t}_{n-2}$.

Case 3. $\mathfrak{t}_{n} \supset \mathfrak{t}_{n-1} \subset \mathfrak{t}_{n-2}$ :

The result follows from case 2 if we switch $\mathfrak{t}_{n-1}$ to $\mathfrak{t}$ in case 2 .

Case 4. $\mathfrak{t}_{n} \subset \mathfrak{t}_{n-1} \subset \mathfrak{t}_{n-2}$ :

Suppose $\mathfrak{t}_{\mathfrak{n}-1}=\mathfrak{t}_{n} \cup\left(l, \nu_{l}\right)$ and $\mathfrak{t}_{n-2}=\mathfrak{t}_{n-1} \cup\left(k, \nu_{k}\right)$ and $\mathfrak{t}_{n-2}=\nu$. If $\mathfrak{t}_{n-1} \triangleleft \mathfrak{t}$, then $k>l$. Let $b=2(f-1)+\sum_{j=1}^{l} \nu_{j}$ and $a=2(f-2)+\sum_{j=1}^{k} \nu_{j}$. Then $a+1 \geq b$. In this situation,

$$
\begin{aligned}
\mathfrak{m}_{\mathfrak{t}} & =e_{2 f-1} s_{2 f, n} s_{2 f-1, b} e_{2 f-3} s_{2 f-2, n-1} s_{2 f-3, a} \mathfrak{m}_{\mathfrak{t}_{n-2}}, \\
\mathfrak{m}_{\mathfrak{t}_{s_{n-1}}} & =e_{2 f-1} s_{2 f, n} s_{2 f-1, a+1} e_{2 f-3} s_{2 f-2, n-1} s_{2 f-3, b-2} \mathfrak{m}_{\mathfrak{t}_{n-2}} .
\end{aligned}
$$

On the other hand, by direct computation (in fact, one can verify the following equalities easily if one uses Brauer diagrams in, e.g. [Wen88]),

$$
\begin{aligned}
& e_{2 f-1} e_{2 f-3} s_{2 f, n} s_{2 f-1, b} s_{2 f-2, n-1} s_{2 f-3, a} s_{n-1} \\
= & e_{2 f-1} e_{2 f-3} s_{2 f-1, n} s_{2 f-2, n-1} s_{2 f-2, a} s_{2 f-3, b-2} \\
= & e_{2 f-1} s_{2 f, n} s_{2 f-1, a+1} e_{2 f-3} s_{2 f-2, n-1} s_{2 f-3, b-2} .
\end{aligned}
$$

Therefore, $\mathfrak{m}_{\mathfrak{t}} s_{n-1}=\mathfrak{m}_{\mathfrak{t} s_{n-1}}$. This completes the proof of (a).

By considering $f_{\mathfrak{t}} s_{k}^{2}=f_{\mathfrak{t}}$ for $\mathfrak{t} s_{k} \triangleleft \mathfrak{t}$, we obtain the result for $\mathfrak{t} s_{k} \triangleright \mathfrak{t}$.

Lemma 3.15. Let $\mathscr{B}_{n}(\delta)$ be a Brauer algebra over a field $F$. Suppose $\mathfrak{t} \in$ $\mathscr{T}_{n}^{u d}(\lambda)$ with $\mathfrak{t}_{k-1} \neq \mathfrak{t}_{k+1}$.

(a) If $\mathfrak{t}_{k} \ominus \mathfrak{t}_{k-1}$ and $\mathfrak{t}_{k} \ominus \mathfrak{t}_{k+1}$ are in the same row, then $f_{\mathfrak{t}} s_{k}=f_{\mathfrak{t}}$.

(b) If $\mathfrak{t}_{k} \ominus \mathfrak{t}_{k-1}$ and $\mathfrak{t}_{k} \ominus \mathfrak{t}_{k+1}$ are in the same column, then $f_{\mathfrak{t}} s_{k}=-f_{\mathfrak{t}}$. 
Proof. Write $f_{\mathfrak{t}} s_{k}=\sum_{\mathfrak{s} \in \mathscr{T}_{n}^{u d}(\lambda)} s_{\mathfrak{t s}}(k) f_{\mathfrak{s}}$. By Lemma 3.11, $\mathfrak{s} \stackrel{k}{\sim} \mathfrak{t}$ if $s_{\mathfrak{t} \mathfrak{s}}(k) \neq 0$. Under the assumption, $\mathfrak{s} \stackrel{k}{\sim} \mathfrak{t}$ if and only if $\mathfrak{s}=\mathfrak{t}$. Therefore, $f_{\mathfrak{t}} s_{k}=s_{\mathfrak{t t}}(k) f_{\mathfrak{t}}$, and $f_{\mathfrak{t}}=s_{\mathfrak{t t}}(k) f_{\mathfrak{t}} s_{k}$. We have $s_{\mathfrak{t t}}(k) \in\{-1,1\}$. Since $f_{\mathfrak{t}} x_{k+1}=f_{\mathfrak{t}} s_{k} x_{k} s_{k}+$ $f_{\mathfrak{t}} s_{k}, s_{\mathfrak{t t}}(k)=1$ (resp. $s_{\mathfrak{t t}}(k)=-1$ ) if $\mathfrak{t}_{k} \ominus \mathfrak{t}_{k-1}$ and $\mathfrak{t}_{k} \ominus \mathfrak{t}_{k+1}$ are in the same row (resp. column).

Lemma 3.16. Let $\mathscr{B}_{n}(\delta)$ be a Brauer algebra over $F$ such that AMR06, 4.12] for $\mathscr{B}_{n}(\delta)$ holds. Suppose that $\mathfrak{t} \in \mathscr{T}_{n}^{u d}(\lambda)$ and $\mathfrak{t}_{k-1}=\mathfrak{t}_{k+1}$. Then

a) $e_{\mathfrak{t t}}(k)=\left(2 c_{\mathfrak{t}}(k)+1\right) \prod_{\substack{\mathfrak{s} \sim \mathfrak{t} \\ \mathfrak{s} \neq \mathfrak{t}}} \frac{c_{\mathfrak{t}}(k)+c_{\mathfrak{s}}(k)}{c_{\mathfrak{t}}(k)-c_{\mathfrak{s}}(k)} \neq 0$.

b) $e_{\mathfrak{t} \mathfrak{s}}(k) e_{\mathfrak{u u}}(k)=e_{\mathfrak{t u}}(k) e_{\mathfrak{u s}}(k)$ for any $\mathfrak{s}, \mathfrak{u} \in \mathscr{T}_{n}^{u d}(\lambda)$ with $\mathfrak{t} \stackrel{k}{\sim} \mathfrak{s} \stackrel{k}{\sim} \mathfrak{u}$.

Proof. Given $(f, \lambda) \in \Lambda_{n}$. We claim $e_{\mathfrak{t t}}(k) \neq 0$ for any $\mathfrak{t} \in \mathscr{T}_{n}^{u d}(\lambda)$ with $\mathfrak{t}_{k-1}=\mathfrak{t}_{k+1}$.

In [AMR06, §4], Ariki, Mathas and the first author constructed the seminormal representations for cyclotomic Nazarov-Wenzl algebras $\mathscr{W}_{r, n}$ under the assumption [AMR06, 4.3] and [AMR06, 4.12], the latter is called the root conditions. In particular, $\mathscr{W}_{1, n}$ is $\mathscr{B}_{n}(\delta)$. In this case, AMR06, 4.3] is our assumption 3.1. Therefore, the seminormal representations $S^{(l, \mu)}$ (denoted by $\Delta(\mu)$ in [AMR06]) for $\mathscr{B}_{n}(\delta)$ were constructed for all $(l, \mu) \in \Lambda_{n}$. More explicitly, each $S^{(l, \mu)}$ has a basis $\left\{v_{\mathfrak{t}} \mid \mathfrak{t} \in \mathscr{T}_{n}^{u d}(\mu)\right\}$ such that

(1) $v_{\mathrm{t}} x_{k}=c_{\mathfrak{t}}(k) v_{\mathrm{t}}$.

(2) $v_{\mathfrak{t}} e_{k}=\sum_{\mathfrak{s} \sim \mathfrak{t}} \tilde{e}_{\mathfrak{t} \mathfrak{s}}(k) v_{\mathfrak{s}}$ with $\tilde{e}_{\mathfrak{t t}}(k) \neq 0$.

Since we are assuming that $F$ satisfies the assumption $3.1, \mathscr{B}_{n}(\delta)$ is semisimple. We have $F v_{\mathfrak{t}}=\cap_{k=1}^{n} S_{c_{\mathfrak{t}}(k)}^{(l, \mu)}$, where $S_{c_{\mathfrak{t}}(k)}^{(l, \mu)}$ is the eigenspace of $S^{(l, \mu)}$ with respect to eigenvalue $c_{\mathfrak{t}}(k)$ of $x_{k}$. Since $\left\{S^{(l, \mu)} \mid(l, \mu) \in \Lambda_{n}\right\}$ consists of all pair-wise non-isomorphic irreducible $\mathscr{B}_{n}(\delta)$-modules when $\mathscr{B}_{n}(\delta)$ is semisimple, $\Delta(f, \lambda) \cong S^{(l, \mu)}$ for some $(l, \mu) \in \Lambda_{n}$. Let $\phi$ be the corresponding isomorphism. Then $\phi\left(f_{\mathfrak{t}}\right) \in \cap_{k=1}^{n} S_{c_{\mathfrak{t}}(k)}^{(l, \mu)}$ for some $(l, \mu) \in \Lambda_{n}$. Therefore, $\phi\left(f_{t}\right)$ is equal to $v_{\mathfrak{s}} \in S^{(l, \mu)}$ for some $\mathfrak{s} \in \mathscr{T}_{n}^{u d}(\mu)$ up to a non-zero scalar. This implies that $e_{\mathfrak{t t}}(k) \neq 0$.

Suppose $k \geq 1$ and that $a \geq 0$. Let $Z\left(\mathscr{B}_{k-1}(\delta)\right)$ be the center of $\mathscr{B}_{k-1}(\delta)$. It is proved in [AMR06, 4.15] that there exist elements $\delta_{k}^{(a)}$ in $Z\left(\mathscr{B}_{k-1}(\delta)\right) \cap$ $F\left[x_{1}, \ldots, x_{k-1}\right]$ such that $e_{k} x_{k}^{a} e_{k}=\delta_{k}^{(a)} e_{k}$. Moreover, the generating series $W_{k}(y)=\sum_{a \geq 0} \delta_{k}^{(a)} y^{-a}$ satisfies

$$
W_{k+1}(y)+y-\frac{1}{2}=\left(W_{k}(y)+y-\frac{1}{2}\right) \frac{\left(y+x_{k}\right)^{2}-1}{\left(y-x_{k}\right)^{2}-1} \cdot \frac{\left(y-x_{k}\right)^{2}}{\left(y+x_{k}\right)^{2}} .
$$

Comparing the coefficient of $f_{\mathfrak{s}}$ on both sides of the identity $f_{\mathfrak{t}} e_{k} W_{k}(y)=$ $f_{\mathrm{t}} e_{k} \frac{y}{y-x_{k}} e_{k}$, we have

$$
W_{k}(y, \mathfrak{s}) y^{-1} e_{\mathfrak{t} \mathfrak{s}}(k)=\sum_{\substack{\mathfrak{s}_{\mathfrak{s}} \sim \mathfrak{t}^{k} \mathfrak{u}\\}} \frac{e_{\mathfrak{t u}}(k) e_{\mathfrak{u s}}(k)}{y-c_{\mathfrak{u}}(k)} .
$$


Therefore,

$$
e_{\mathfrak{t s}}(k) \cdot \operatorname{Res}_{y=c_{\mathfrak{u}}(k)} W_{k}(y, \mathfrak{s}) y^{-1}=e_{\mathfrak{u s}}(k) e_{\mathfrak{t u}}(k) .
$$

Since $e_{\mathfrak{t t}}(k) \neq 0, e_{\mathfrak{t t}}(k)=\operatorname{Res}_{y=c_{\mathfrak{t}}(k)} W_{k}(y, \mathfrak{t}) y^{-1}$ by assuming that $\mathfrak{t}=\mathfrak{s}=\mathfrak{u}$.

If $\mathfrak{s} \stackrel{k}{\sim} \mathfrak{u}$, then $c_{\mathfrak{s}}(j)=c_{\mathfrak{u}}(j)$ for $j \leq k-1$. Note that $W_{k}(y) \in$ $F\left[x_{1}, x_{2}, \cdots, x_{k-1}\right], \quad W_{k}(y, \mathfrak{s})=W_{k}(y, \mathfrak{u}) . \quad$ Therefore, $e_{\mathfrak{t s}}(k) e_{\mathfrak{u u}}(k)=$ $e_{\mathfrak{u} \mathfrak{s}}(k) e_{\mathfrak{t u}}(k)$. In order to prove (a), we need to prove

$$
\operatorname{Res}_{y=c_{\mathfrak{t}}(k)} W_{k}(y, \mathfrak{t}) / y=\left(2 c_{\mathfrak{t}}(k)+1\right) \prod_{\substack{\mathfrak{s} \sim \mathfrak{t} \\ \mathfrak{s} \neq \mathfrak{t}}} \frac{c_{\mathfrak{t}}(k)+c_{\mathfrak{s}}(k)}{c_{\mathfrak{t}}(k)-c_{\mathfrak{s}}(k)},
$$

which is [AMR06, 4.8] for $r=1$.

It is proved in [AMR06, 5.4] that the real field $\mathbb{R}$ satisfies the assumption 3.1 and AMR06, 4.12]. If we do not use Lemma 3.16(a), we can remove the assumption [AMR06, 4.12].

Lemma 3.17. Let $\mathscr{B}_{n}(\delta)$ be a Brauer algebra over a field $F$. Suppose $\mathfrak{t} \in$ $\mathscr{T}_{n}^{u d}(\lambda)$ with $\mathfrak{t}_{k-1}=\mathfrak{t}_{k+1}$.

a) $f_{\mathfrak{t}} e_{k}=\sum_{\mathfrak{s} \sim \mathfrak{t}} e_{\mathfrak{t} \mathfrak{s}}(k) f_{\mathfrak{s}}$. Furthermore, $\left\langle f_{\mathfrak{s}}, f_{\mathfrak{s}}\right\rangle e_{\mathfrak{t} \mathfrak{s}}(k)=\left\langle f_{\mathfrak{t}}, f_{\mathfrak{t}}\right\rangle e_{\mathfrak{s t}}(k)$.

b) $f_{\mathfrak{t}} s_{k}=\sum_{\mathfrak{s} \sim \mathfrak{t}} s_{\mathfrak{t s}}(k) f_{\mathfrak{s}}$. Furthermore, $s_{\mathfrak{t s}}(k)=\frac{e_{\mathfrak{t} \mathfrak{s}}(k)-\delta_{\mathfrak{t s}}}{c_{\mathfrak{t}}(k)+c_{\mathfrak{s}}(k)}$.

Proof. (a) follows from $\left\langle f_{\mathfrak{t}} e_{k}, f_{\mathfrak{s}}\right\rangle=\left\langle f_{\mathfrak{t}}, f_{\mathfrak{s}} e_{k}\right\rangle$, and $\left\langle f_{\mathfrak{s}}, f_{\mathfrak{t}}\right\rangle=\delta_{\mathfrak{s t}}\left\langle f_{\mathfrak{t}}, f_{\mathfrak{t}}\right\rangle$. (b) follows from (a) and Lemma 2.10(d).

We organise the above results as follows. Such a result can be considered as a generalisation of Dipper-James theorem for Hecke algebras of type $A$ DJ86.

Theorem 3.18. Let $\mathscr{B}_{n}(\delta)$ be a Brauer algebra over a field $F$. Assume that $\mathfrak{t} \in \mathscr{T}_{n}^{u d}(\lambda)$.

a) Suppose $\mathfrak{t}_{k-1} \neq \mathfrak{t}_{k+1}$. Then

$f_{\mathfrak{t}} s_{k}= \begin{cases}f_{\mathfrak{t}}, & \text { if } \mathfrak{t}_{k} \ominus \mathfrak{t}_{k-1} \text { and } \mathfrak{t}_{k} \ominus \mathfrak{t}_{k+1} \text { are in the same row of } \mathfrak{t}_{k}, \\ -f_{t}, & \text { if } \mathfrak{t}_{k} \ominus \mathfrak{t}_{k-1} \text { and } \mathfrak{t}_{k} \ominus \mathfrak{t}_{k_{1+1}} \text { are in the same column of } \mathfrak{t}_{k}, \\ \frac{1}{c_{\mathfrak{t}}(k+1)-c_{\mathfrak{t}}(k)} f_{\mathfrak{t}}+f_{\mathfrak{t}_{k}}, & \text { if } \mathfrak{t}_{k} \in \mathscr{T}_{n}^{\text {ud }}(\lambda) \text { and } \mathfrak{t}_{k} \triangleleft \mathfrak{t}, \\ \frac{1}{c_{\mathfrak{t}}(k+1)-c_{\mathfrak{t}}(k)} f_{\mathfrak{t}}+c f_{\mathfrak{t}_{k}}, & \text { if } \mathfrak{t}_{k} \in \mathscr{T}_{n}^{\text {ud }}(\lambda) \text { and } \mathfrak{t}_{k} \triangleright \mathfrak{t},\end{cases}$ where $c=\frac{\left(c_{\mathrm{t}}(k+1)-c_{\mathrm{t}}(k)+1\right)\left(c_{\mathrm{t}}(k+1)-c_{\mathrm{t}}(k)-1\right)}{\left(c_{\mathrm{t}}(k+1)-c_{\mathrm{t}}(k)\right)^{2}}$.

b) Suppose $\mathfrak{t}_{k-1} \neq \mathfrak{t}_{k+1}$. Then $f_{\mathfrak{t}} e_{k}=0$.

c) Suppose $\mathfrak{t}_{k-1}=\mathfrak{t}_{k+1}$. Then $f_{\mathfrak{t}} e_{k}=\sum_{\mathfrak{s} \sim \mathfrak{t}} e_{\mathfrak{t} \mathfrak{s}}(k) f_{\mathfrak{s}}$ with

$$
\left\langle f_{\mathfrak{s}}, f_{\mathfrak{s}}\right\rangle e_{\mathfrak{t} \mathfrak{s}}(k)=\left\langle f_{\mathfrak{t}}, f_{\mathfrak{t}}\right\rangle e_{\mathfrak{s t}}(k) .
$$

d) Suppose $\mathfrak{t}_{k-1}=\mathfrak{t}_{k+1}$. Then $f_{\mathfrak{t}} s_{k}=\sum_{\mathfrak{s} \sim \mathfrak{t}} s_{\mathfrak{t s}}(k) f_{\mathfrak{s}}$ with

$$
s_{\mathfrak{t \mathfrak { s }}}(k)=\frac{e_{\mathfrak{t} \mathfrak{s}}(k)-\delta_{\mathrm{t} \mathfrak{s}}}{c_{\mathfrak{t}}(k)+c_{\mathfrak{s}}(k)} \text {. }
$$


The following result gives an explicit construction on primitive idempotents and central primitive idempotents for $\mathscr{B}_{n}(\delta)$. This gives the Wedderburn-Artin decomposition for Brauer algebras $\mathscr{B}_{n}(\delta)$. Such results can be proved by the arguments in [Mat04]. In [Mat06, 3.16], Mathas proved this result for a class of cellular algebras satisfying a "separated condition", which is analogue to the assumption 3.1. In our case, such idempotents can be computed explicitly since, in the next section, we give recursive formulae for $\left\langle f_{\mathfrak{t}}, f_{\mathfrak{t}}\right\rangle$ for all $\mathfrak{t} \in \mathscr{T}_{n}^{u d}(\lambda)$ and all $(f, \lambda) \in \Lambda_{n}$.

Proposition 3.19. Let $\mathscr{B}_{n}(\delta)$ be a Brauer algebra over a field $F$.

a) Suppose $\mathfrak{t} \in \mathscr{T}_{n}^{u d}(\lambda)$. Then $\frac{1}{\left\langle f_{\mathfrak{t}}, f_{\mathfrak{t}}\right\rangle} f_{\mathfrak{t t}}$ is a primitive idempotent of $\mathscr{B}_{n}(\delta)$ with respect to the cell module $\Delta(f, \lambda)$.

b) $\sum_{\mathfrak{t} \in \mathscr{T}_{n}^{u d}(\lambda)} \frac{1}{\left\langle f_{\mathfrak{t}}, f_{\mathfrak{t}}\right\rangle} f_{\mathfrak{t t}}$ is a central primitive idempotent. Furthermore,

$$
\sum_{\left(\frac{n-|\lambda|}{2}, \lambda\right) \in \Lambda_{n}} \sum_{\mathfrak{t} \in \mathscr{T}_{n}^{u d}(\lambda)} \frac{1}{\left\langle f_{\mathfrak{t}}, f_{\mathfrak{t}}\right\rangle} f_{\mathfrak{t t}}=1 .
$$

\section{Discriminants of Gram matrices}

In this section, we compute Gram determinants associated to all cell modules of $\mathscr{B}_{n}(\delta)$. Unless otherwise stated, we assume that $F$ is a field which satisfies the assumption 3.1. When we use Lemma 3.16)(a) in Proposition 4.8 4.9, we have to assume that [AMR06, 4.12] for $\mathscr{B}_{n}(\delta)$ holds.

Gram determinants for all cell modules for Hecke algebras of type $A$ were computed by Dipper and James in [DJ86]. When $q=1$, they are the formulae for symmetric groups $\mathfrak{S}_{n}$. Therefore, our formulae for $\operatorname{det} G_{0, \lambda}$ are special cases of those formulae.

Definition 4.1. Given $\mathfrak{s} \in \mathscr{T}_{n}^{u d}(\lambda)$ with $\mathfrak{s}_{n-1}=\mu$, define

a) $\hat{\mathfrak{s}} \in \mathscr{T}_{n-1}^{u d}(\mu)$, such that $\hat{\mathfrak{s}}_{j}=\mathfrak{s}_{j}$, for all $1 \leq j \leq n-1$,

b) $\tilde{\mathfrak{s}} \in \mathscr{T}_{n}^{u d}(\lambda)$ such that $\tilde{\mathfrak{s}}_{i}=\mathfrak{t}_{i}^{\mu}, 1 \leq i \leq n-1$ and $\tilde{\mathfrak{s}}_{n}=\lambda$.

For any partition $\lambda=\left(\lambda_{1}, \lambda_{2}, \cdots,\right)$, let $\lambda !=\prod_{i} \lambda_{i} ! \in \mathbb{N}$. For any $\mathfrak{t} \in$ $\mathscr{T}_{n}^{u d}(\lambda)$, define

$$
F_{\mathfrak{t}, k}=\prod_{\substack{r \in \mathscr{R}(k) \\ r \neq c_{\mathfrak{t}}(k)}} \frac{x_{k}-r}{c_{\mathfrak{t}}(k)-r}, \quad 1 \leq k \leq n .
$$

Proposition 4.2. Assume that $\mathfrak{t} \in \mathscr{T}_{n}^{u d}(\lambda)$ with $(f, \lambda) \in \Lambda_{n}$. If $\mathfrak{t}_{n-1}=\mu$ with $(l, \mu) \in \Lambda_{n-1}$, then $\left\langle f_{\mathfrak{t}}, f_{\mathfrak{t}}\right\rangle=\frac{1}{\delta^{l} \mu !}\left\langle f_{\hat{\mathfrak{t}}}, f_{\hat{\mathfrak{t}}}\right\rangle\left\langle f_{\tilde{\mathfrak{t}}}, f_{\tilde{\mathfrak{t}}}\right\rangle$.

Proof. By the definition of $F_{\mathfrak{t}}$, we have $F_{\mathfrak{t}}=F_{\hat{\mathfrak{t}}} F_{\mathfrak{t}, n}$. Using the definition of $\mathfrak{m}_{\mathfrak{t}}$, we have

$$
f_{\mathfrak{t}^{\lambda} \mathfrak{t}}=F_{\mathfrak{t}^{\lambda}} h \mathfrak{m}_{\mathfrak{t}^{\mu}, \hat{\mathfrak{t}}} F_{\hat{\mathfrak{t}}} F_{\mathfrak{t}, n}
$$

for some $h \in \mathscr{B}_{n}(\delta)$. Note that

$$
\mathfrak{m}_{\mathfrak{t}^{\mu}, \hat{\mathfrak{t}}} F_{\hat{\mathfrak{t}}} F_{\hat{\mathfrak{t}}} \mathfrak{m}_{\hat{\mathfrak{t}}, \mathfrak{t}^{\mu}} \equiv\left\langle f_{\hat{\mathfrak{t}}}, f_{\hat{\mathfrak{t}}}\right\rangle \mathfrak{m}_{\mathfrak{t}^{\mu}, \mathfrak{t}^{\mu}} \quad\left(\bmod \mathscr{B}_{n-1}(\delta)^{\triangleright(l, \mu)}\right) .
$$


By Theorem 2.5 (c) and Theorem 2.11 and Corollary 3.8,

$$
\begin{aligned}
& f_{\mathfrak{t}^{\lambda} \mathfrak{t}} f_{\mathfrak{t t}^{\lambda}} \equiv F_{\mathfrak{t}^{\lambda}} \mathfrak{m}_{\mathfrak{t}} F_{\hat{\mathfrak{t}}} F_{\hat{\mathfrak{t}}} \mathfrak{m}_{\hat{\mathfrak{t}}, \mu^{\mu}} F_{\mathfrak{t}, n}^{2} \sigma(h) F_{\mathfrak{t}^{\lambda}} \quad\left(\bmod \mathscr{B}_{n}(\delta)^{\triangleright(f, \lambda)}\right) \\
& \equiv\left\langle f_{\hat{\mathfrak{t}}}, f_{\hat{\mathfrak{t}}}\right\rangle F_{\mathfrak{t}^{\lambda}} \mathfrak{m}_{\mathfrak{t}^{\lambda}, \tilde{\mathfrak{t}}} F_{\mathfrak{t}, n}^{2} \sigma(h) F_{\mathfrak{t}^{\lambda}} \quad\left(\bmod \mathscr{B}_{n}(\delta)^{\triangleright(f, \lambda)}\right) \\
& \equiv \frac{\left\langle f_{\hat{\mathfrak{t}}}, f_{\hat{\mathfrak{t}}}\right\rangle}{\delta^{l} \mu !} F_{\mathfrak{t}^{\lambda}} h F_{\mathfrak{t}, n} \mathfrak{m}_{\mu} F_{\mathfrak{t}^{\mu}} F_{\mathfrak{t}^{\mu}} \mathfrak{m}_{\mu} F_{\mathfrak{t}, n} \sigma(h) F_{\mathfrak{t}^{\lambda}} \quad\left(\bmod \mathscr{B}_{n}(\delta)^{\triangleright(f, \lambda)}\right) \\
& \equiv \frac{\left\langle f_{\hat{\mathfrak{t}}}, f_{\hat{\mathfrak{t}}}\right\rangle}{\delta^{l} \mu !} f_{\mathfrak{t}^{\lambda} \hat{\mathfrak{t}}} f_{\tilde{\mathfrak{t}}^{\lambda}} \quad\left(\bmod \mathscr{B}_{n}(\delta)^{\triangleright(f, \lambda)}\right) \\
& \equiv \frac{\left\langle f_{\hat{\mathfrak{t}}}, f_{\hat{\mathfrak{t}}}\right\rangle\left\langle f_{\tilde{\mathfrak{t}}}, f_{\tilde{\mathfrak{t}}}\right\rangle}{\delta^{l} \mu !} f_{\mathfrak{t}^{\lambda} \mathfrak{t}^{\lambda}} \quad\left(\bmod \mathscr{B}_{n}(\delta)^{\triangleright(f, \lambda)}\right) .
\end{aligned}
$$

Consequently, we have the formula for $\left\langle f_{\mathfrak{t}}, f_{\mathfrak{t}}\right\rangle$, as required.

Lemma 4.3. Assume $\mathfrak{t} \in \mathscr{T}_{n}^{u d}(\lambda)$ with $(f, \lambda) \in \Lambda_{n}$. If $\mathfrak{t}_{k} \in \mathscr{T}_{n}^{u d}(\lambda)$ with $\mathfrak{t} s_{k} \triangleleft \mathfrak{t}$, then $\left\langle f_{\mathfrak{t s}_{k}}, f_{\mathfrak{t s}_{k}}\right\rangle=\left(1-\left(c_{\mathfrak{t}}(k+1)-c_{\mathfrak{t}}(k)\right)^{-2}\right)\left\langle f_{\mathfrak{t}}, f_{\mathfrak{t}}\right\rangle$.

Proof. The result follows from Lemma 3.14 and $\left\langle f_{\mathfrak{t}}, f_{\mathfrak{t}}\right\rangle=\left\langle f_{\mathfrak{t}} s_{k}, f_{\mathfrak{t}} s_{k}\right\rangle$.

Definition 4.4. For any $\lambda \vdash n-2 f$, let $\mathscr{A}(\lambda)$ (resp. $\mathscr{R}(\lambda)$ ) be the set of all addable (resp. removable) nodes of $\lambda$. Given a removable (resp. an addable) node $p=\left(k, \lambda_{k}\right)$ (resp. $\left.\left(k, \lambda_{k}+1\right)\right)$ of $\lambda$, define

a) $\mathscr{R}(\lambda)^{<p}=\left\{\left(l, \lambda_{l}\right) \in \mathscr{R}(\lambda) \mid l>k\right\}$,

b) $\mathscr{A}(\lambda)^{<p}=\left\{\left(l, \lambda_{l}+1\right) \in \mathscr{A}(\lambda) \mid l>k\right\}$,

c) $\mathscr{A} \mathscr{R}(\lambda)^{\geq p}=\left\{\left(l, \lambda_{l}\right) \in \mathscr{R}(\lambda) \mid l \leq k\right\} \cup\left\{\left(l, \lambda_{l}+1\right) \in \mathscr{A}(\lambda) \mid l \leq k\right\}$.

Proposition 4.5. Suppose $\mathfrak{t} \in \mathscr{T}_{n}^{u d}(\lambda)$ with $\lambda \vdash n-2 f$. If $\hat{\mathfrak{t}}=\mathfrak{t}^{\mu}$ and $\mathfrak{t}_{n}=\mathfrak{t}_{n-1} \cup\{p\}$ with $p=\left(k, \lambda_{k}\right)$, then

$$
\left\langle f_{\mathfrak{t}}, f_{\mathfrak{t}}\right\rangle=-\delta^{f} \mu ! \frac{\prod_{q \in \mathscr{A}(\lambda)<p}\left(c_{\lambda}(p)+c_{\lambda}(q)\right)}{\prod_{r \in \mathscr{R}(\lambda)<p}\left(c_{\lambda}(p)-c_{\lambda}(r)\right)} .
$$

Proof. By assumption, $\mathfrak{t}=\mathfrak{t}^{\lambda} s_{a_{k}, n}$, where $a_{k}=2 f+\sum_{i=1}^{k} \lambda_{i}$. Using Lemma 4.3 repeatedly for the pairs $\left\{f_{\mathfrak{t}^{\lambda} s_{a_{k}, j}}, f_{\mathfrak{t}^{\lambda} s_{a_{k}, j+1}}\right\}$ with $a_{k} \leq j \leq n-1$, and noting that $\mathfrak{t} \triangleleft \mathfrak{t} s_{n-1} \cdots \triangleleft \mathfrak{t} s_{n, a_{k}}=\mathfrak{t}^{\lambda}$, we have

$$
\left\langle f_{\mathfrak{t}}, f_{\mathfrak{t}}\right\rangle=\left\langle f_{\mathfrak{t}^{\lambda}}, f_{\mathfrak{t}^{\lambda}}\right\rangle \prod_{j=a_{k}}^{n-1}\left(1-\left(c_{\mathfrak{t}}(n)-c_{\mathfrak{t}}(j)\right)^{-2}\right) .
$$

Since $f_{\mathfrak{t}^{\lambda}} \equiv \mathfrak{m}_{\lambda}\left(\bmod \mathscr{B}_{n}(\delta)^{\triangleright(f, \lambda)}\right),\left\langle f_{\mathfrak{t}^{\lambda}}, f_{\mathfrak{t}^{\lambda}}\right\rangle=\delta^{f} \lambda$ !. If $a, b \in \mathbb{Z}$ with $b>a$, then

$$
\prod_{i=a}^{b} \frac{(q-i)^{2}-1}{(q-i)^{2}}=\frac{q-a+1}{q-a} \frac{q-b-1}{q-b}
$$

We compute $\prod_{j=a_{k}}^{n-1}\left(1-\left(c_{\mathfrak{t}}(n)-c_{\mathfrak{t}}(j)\right)^{-2}\right)$ along each row of $\mathbf{t}^{\lambda}$ first. Via (4.7), we need only consider the first and the last nodes in each row. Note that $c_{\lambda}(p)=-c_{\mathfrak{t}}(n)$. By (4.7) again, we have

$$
\prod_{j=a_{k}}^{n-1}\left(1-\left(c_{\mathfrak{t}}(n)-c_{\mathfrak{t}}(j)\right)^{-2}\right)=-\frac{1}{\lambda_{k}} \frac{\prod_{q \in \mathscr{A}(\lambda)<p}\left(c_{\lambda}(p)+c_{\lambda}(q)\right)}{\prod_{r \in \mathscr{R}(\lambda)<p}\left(c_{\lambda}(p)-c_{\lambda}(r)\right)},
$$


proving (4.6).

Proposition 4.8. Suppose $\mathfrak{t} \in \mathscr{T}_{n}^{u d}(\lambda)$ with $\lambda=\left(\lambda_{1}, \ldots, \lambda_{k}\right) \vdash n-2 f$. If $\mathfrak{t}^{\mu}=\hat{\mathfrak{t}}$ and $\mathfrak{t}_{n-1}=\mathfrak{t}_{n} \cup p$ with $p=\left(k, \mu_{k}\right)$, then

$$
\left\langle f_{\mathfrak{t}}, f_{\mathfrak{t}}\right\rangle=A \cdot \delta^{f-1} \mu ! \cdot\left(\delta+2 \mu_{k}-2 k\right) \prod_{\substack{q \neq p \\ q \in \mathscr{A} \mathscr{R}(\lambda) \geq p}} \frac{c_{\lambda}(p)+c_{\lambda}(q)}{c_{\lambda}(p)-c_{\lambda}(q)}
$$

where $A=1$ if $\lambda_{k}=0$ and $A=\delta+\lambda_{k}-2 k$ if $\lambda_{k}>0$.

Proof. Let $a=2(f-1)+\sum_{j=1}^{k-1} \mu_{j}+1$. Write $\tilde{\mathfrak{m}}_{\lambda}=\mathfrak{m}_{\mathfrak{t}^{\lambda}}$ for $\mathfrak{t}^{\lambda} \in \mathscr{T}_{n-2}^{u d}(\lambda)$. We have

$$
\begin{aligned}
f_{\mathfrak{t}} e_{n-1} & \equiv e_{2 f-1} s_{2 f, n} s_{2 f-1, n-1} \tilde{\mathfrak{m}}_{\lambda} \sum_{j=a}^{n-1} s_{n-1, j} F_{\mathfrak{t}} e_{n-1} \quad\left(\bmod \mathscr{B}_{n}(\delta)^{\triangleright(f, \lambda)}\right) \\
& \equiv e_{2 f-1} s_{2 f, n} s_{2 f-1, n-1} \tilde{\mathfrak{m}}_{\lambda} \sum_{j=a}^{n-1} s_{n-1, j} F_{\mathfrak{t}, n-1} F_{\mathfrak{t}, n} e_{n-1} \prod_{k=1}^{n-2} F_{\mathfrak{t}, k} \quad\left(\bmod \mathscr{B}_{n}(\delta)^{\triangleright(f, \lambda)}\right)
\end{aligned}
$$

Since $e_{n-1} x_{n-1}^{k} e_{n-1}=\delta_{n-1}^{(k)}$ for some $\delta_{n-1}^{(k)} \in F\left[x_{1}, x_{2}, \cdots, x_{n-2}\right] \cap$ $Z\left(\mathscr{B}_{n-2}(\delta)\right)$,

$$
e_{n-1} F_{\mathfrak{t}, n-1} F_{\mathfrak{t}, n} e_{n-1}=\Phi_{\mathfrak{t}}\left(x_{1}, \cdots, x_{n-2}\right) e_{n-1},
$$

for some $\Phi_{\mathfrak{t}}\left(x_{1}, \cdots, x_{n-2}\right) \in F\left[x_{1}, x_{2}, \cdots, x_{n-2}\right] \cap Z\left(\mathscr{B}_{n-2}(\delta)\right)$. In Naz96, 2.4], Nazarov proved that

$$
x_{k}^{i} s_{k}=s_{k} x_{k+1}^{i}+\sum_{j=1}^{i} x_{k}^{i-j}\left(e_{k}-1\right) x_{k+1}^{j-1}
$$

for $\mathscr{B}_{n}(\delta)$ over $\mathbb{C}$. In fact, it holds for $\mathscr{B}_{n}(\delta)$ over $F$ [AMR06, 2.3]. Therefore, using the above equality for $k=n-2$, we have

$$
e_{n-1} s_{n-2} F_{\mathfrak{t}, n-1} F_{\mathfrak{t}, n} e_{n-1}=\Psi_{\mathfrak{t}}\left(x_{1}, \cdots, x_{n-2}\right) e_{n-1}
$$

for some $\Psi_{\mathfrak{t}}\left(x_{1}, \cdots, x_{n-2}\right) \in F\left[x_{1}, x_{2}, \cdots, x_{n-2}\right]$. In order to simplify the notation, we use $\Phi_{\mathfrak{t}}$ and $\Psi_{\mathfrak{t}}$ instead of $\Phi_{\mathfrak{t}}\left(x_{1}, \cdots, x_{n-2}\right)$ and $\Psi_{\mathfrak{t}}\left(x_{1}, \cdots, x_{n-2}\right)$ respectively. Let $\Phi_{\mathfrak{t}, \lambda}$ (resp. $\left.\Psi_{\mathfrak{t}, \lambda}\right)$ be obtained by using $c_{\mathfrak{t}^{\lambda}}(k)$ instead of $x_{k}$ in $\Phi_{\mathfrak{t}}\left(\operatorname{resp} . \Psi_{\mathfrak{t}}\right)$. Then

$$
\begin{aligned}
e_{2 f-1} \cdots e_{n-2} \tilde{\mathfrak{m}}_{\lambda} \Phi_{\mathfrak{t}} e_{n-1} \equiv \Phi_{\mathfrak{t}, \lambda} \mathfrak{m}_{\mathfrak{u}} & +\sum_{\substack{\mathfrak{v} \in \mathscr{T}_{1}^{u d}(\lambda) \\
\mathfrak{v}_{n-1}=\mathfrak{u}_{n-1} \\
\mathfrak{v}_{n-2} \triangleright \mathfrak{u}_{n-2}}} b_{\mathfrak{v}} \mathfrak{m}_{\mathfrak{v}} \quad\left(\bmod \mathscr{B}_{n}(\delta)^{\triangleright(f, \lambda)}\right) \\
e_{2 f-1} \cdots e_{n-2} \tilde{\mathfrak{m}}_{\lambda} \Psi_{\mathfrak{t}} e_{n-1} \sum_{j=a}^{n-2} s_{n-2, j} \equiv & \left(\mu_{k}-1\right) \Psi_{\mathfrak{t}, \lambda} \mathfrak{m}_{\mathfrak{u}} \\
& +\sum_{\substack{\mathfrak{v} \in \mathscr{T}_{n} u d \\
\text { val } \\
\mathfrak{v}_{n-1}=\mathfrak{u}_{n-1} \\
\mathfrak{v}_{n-2} \triangleright \mathfrak{u}_{n-2}}} a_{\mathfrak{v}} \mathfrak{m}_{\mathfrak{v}} \quad\left(\bmod \mathscr{B}_{n}(\delta)^{\triangleright(f, \lambda)}\right),
\end{aligned}
$$


where $\mathfrak{u} \stackrel{n-1}{\sim} \mathfrak{t}$ with

$$
\mathfrak{u}_{n-1}= \begin{cases}\mathfrak{t}_{n-2} \cup\{(k+1,1)\}, & \text { if } \mu_{k}>1, \\ \mathfrak{t}_{n-1}, & \text { if } \mu_{k}=1 .\end{cases}
$$

We remark that $\mathfrak{m}_{\mathfrak{u}}=e_{2 f-1} \cdots e_{n-2} e_{n-1} \tilde{\mathfrak{m}}_{\lambda}$ and $\mathfrak{u} \triangleleft \mathfrak{v}$ for any $\mathfrak{v} \in \mathscr{T}_{n}^{u d}(\lambda)$ with $\mathfrak{v} \stackrel{n-1}{\sim} \mathfrak{t}$. Therefore,

$$
f_{\mathfrak{t}} e_{n-1} \equiv\left(\Phi_{\mathfrak{t}, \lambda}+\left(\mu_{k}-1\right) \Psi_{\mathfrak{t}, \lambda}\right) \mathfrak{m}_{\mathfrak{u}}+\sum_{\substack{\mathfrak{v} \in \mathscr{T}_{u}^{u d}(\lambda) \\ \mathfrak{v}_{\mathfrak{n}}=1 \\ \mathfrak{v}_{n-2} \triangleright \mathfrak{u}_{n-1}}}\left(a_{\mathfrak{v}}+b_{\mathfrak{v}}\right) \mathfrak{m}_{\mathfrak{v}} \prod_{k=1}^{n-2} F_{\mathfrak{t}, k} \quad\left(\bmod \mathscr{B}_{n}(\delta)^{\triangleright(f, \lambda)}\right)
$$

In particular, $e_{\mathfrak{t u}}(n-1)=\Phi_{\mathfrak{t}, \lambda}+\left(\mu_{k}-1\right) \Psi_{\mathfrak{t}, \lambda}$. On the other hand, we have

$$
\begin{aligned}
\Phi_{\mathfrak{t}, \lambda} f_{\mathfrak{t}} e_{n-1} & \equiv f_{\mathfrak{t}} e_{n-1} F_{\mathfrak{t}, n-1} F_{\mathfrak{t}, n} e_{n-1} \quad\left(\bmod \mathscr{B}_{n}(\delta)^{\triangleright(f, \lambda)}\right) \\
& \equiv \sum_{\substack{n-1 \\
\mathfrak{v}^{n} \mathfrak{t}}} e_{\mathfrak{t}}(n-1) f_{\mathfrak{v}} F_{\mathfrak{t}, n-1} F_{\mathfrak{t}, n} e_{n-1} \quad\left(\bmod \mathscr{B}_{n}(\delta)^{\triangleright(f, \lambda)}\right) \\
& \equiv e_{\mathfrak{t t}}(n-1) f_{\mathfrak{t}} e_{n-1} \quad\left(\bmod \mathscr{B}_{n}(\delta)^{\triangleright(f, \lambda)}\right) .
\end{aligned}
$$

Since we are assuming that [AMR06, 4.12] holds, $e_{\mathfrak{t t}}(n-1) \neq 0$. So $f_{\mathfrak{t}} e_{n-1} \neq$ 0 and $e_{\mathfrak{t t}}(n-1)=\Phi_{\mathfrak{t}, \lambda}$. If $\mu_{k}=1$, then $\left(\mu_{k}-1\right) \Psi_{\mathfrak{t}, \lambda}=0$. Suppose $\mu_{k}>1$. Then $s_{n-2} \in \mathfrak{S}_{\mu}$. If we use $\Psi_{\mathfrak{t}, \lambda}$ instead of $\Phi_{\mathfrak{t}, \lambda}$ above, we obtain $e_{\mathfrak{t t}}(n-1)=\Psi_{\mathfrak{t}, \lambda}$. In this case, $f_{\mathfrak{t}} s_{n-2}=f_{\mathfrak{t}}$ since both $\mathfrak{t}_{n-1} \ominus \mathfrak{t}_{n-2}$ and $\mathfrak{t}_{n-2} \ominus \mathfrak{t}_{n-3}$ are in the same row. Therefore,

$$
\mu_{k} e_{\mathfrak{t t}}(n-1)=\Phi_{\mathfrak{t}, \lambda}+\left(\mu_{k}-1\right) \Psi_{\mathfrak{t}, \lambda}=e_{\mathfrak{t u}}(n-1) .
$$

Now, we compute $\left\langle f_{\mathfrak{u}}, f_{\mathfrak{u}}\right\rangle$. By the similar argument as above, we have $f_{\mathfrak{t}^{\lambda} \mathfrak{u}} f_{\mathfrak{u t}^{\lambda}}$

$\equiv F_{\mathfrak{t} \lambda} e_{2 f-1} s_{2 f, n} s_{2 f-1, n-1} \tilde{\mathfrak{m}}_{\lambda} F_{\mathfrak{u}, n-1} F_{\mathfrak{u}, n} \tilde{\mathfrak{m}}_{\lambda} s_{n-1,2 f-1} s_{n, 2 f} e_{2 f-1} F_{\mathfrak{t}^{\lambda}} \quad\left(\bmod \mathscr{B}_{n}(\delta)^{\triangleright(f, \lambda)}\right)$ $\equiv F_{\mathfrak{t}^{\lambda}} e_{2 f-1} \cdots e_{n-2} \tilde{\mathfrak{m}}_{\lambda} e_{n-1} F_{\mathfrak{u}, n-1} F_{\mathfrak{u}, n} e_{n-1} \tilde{\mathfrak{m}}_{\lambda} e_{n-2} \cdots e_{2 f-1} F_{\mathfrak{t}^{\lambda}} \quad\left(\bmod \mathscr{B}_{n}(\delta)^{\triangleright(f, \lambda)}\right)$ $\equiv F_{\mathbf{t}^{\lambda}} e_{2 f-1} \cdots e_{n-2} \tilde{\mathfrak{m}}_{\lambda} e_{n-1} \Phi_{\mathfrak{u}^{1}} \tilde{\mathfrak{m}}_{\lambda} e_{n-2} \cdots e_{2 f-1} F_{\mathfrak{t}^{\lambda}} \quad\left(\bmod \mathscr{B}_{n}(\delta)^{\triangleright(f, \lambda)}\right)$ $\equiv \delta^{f-1} \lambda ! F_{\mathfrak{t}^{\lambda}} e_{2 f-1} \cdots e_{n-2} e_{n-1} \tilde{\mathfrak{m}}_{\lambda} \Phi_{\mathfrak{u}} e_{n-2} \cdots e_{2 f-1} F_{\mathfrak{t}^{\lambda}} \quad\left(\bmod \mathscr{B}_{n}(\delta)^{\triangleright(f, \lambda)}\right)$

Note that $\Phi_{\mathfrak{u}} \in F\left[x_{1}, \cdots, x_{n-2}\right]$. By Lemma 3.6 and Corollary 3.8,

$$
\begin{aligned}
& F_{\mathfrak{t}^{\lambda}} e_{2 f-1} \cdots e_{n-2} e_{n-1} \tilde{\mathfrak{m}}_{\lambda} \Phi_{\mathfrak{u}} \\
& \equiv \Phi_{\mathfrak{u}, \lambda} F_{\mathfrak{t}^{\lambda}} e_{2 f-1} \cdots e_{n-2} e_{n-1} \tilde{\mathfrak{m}}_{\lambda}+\sum_{\substack{\mathfrak{v} \in \mathscr{T}^{u d}(\lambda) \\
\mathfrak{v}_{n-1}=\mathfrak{u}_{n-1} \\
\mathfrak{v}_{n-2} \triangleright \mathfrak{u}_{n-2}}} a_{\mathfrak{v}} F_{\mathfrak{t}^{\lambda}} f_{\mathfrak{v}} \quad\left(\bmod \mathscr{B}_{n}(\delta)^{\triangleright(f, \lambda)}\right)
\end{aligned}
$$

By Lemma 3.11, $f_{\mathfrak{v}} e_{n-2} \cdots e_{2 f-1}\left(\bmod \mathscr{B}_{n}(\delta)^{\triangleright(f, \lambda)}\right)$ can be written as a linear combination of $f_{\mathfrak{s}}$ with $\mathfrak{s}_{n-1}=\mathfrak{v}_{\mathfrak{n}-1}$ which is not equal to $\left(\mathfrak{t}^{\lambda}\right)_{n-1}$ under the assumption $\mathfrak{t}_{n-2}=\lambda \neq \varnothing$. If $\mathfrak{t}_{n-2}=\varnothing$, then there is no $\mathfrak{v} \in \mathscr{T}_{n}^{u d}(\lambda)$ such that $\mathfrak{v}_{n-2} \triangleright \mathfrak{u}_{n-2}$. In any case, for $\mathfrak{v} \in \mathscr{T}_{n}^{u d}(\lambda), \mathfrak{v}_{n-1}=\mathfrak{u}_{n-1}$ and $\mathfrak{v}_{n-2} \triangleright \mathfrak{u}_{n-2}$, we have, by Lemma 3.9, that

$$
f_{\mathfrak{v}} e_{n-2} \cdots e_{2 f-1} F_{\mathfrak{t}^{\lambda}} \equiv 0 \quad\left(\bmod \mathscr{B}_{n}(\delta)^{\triangleright(f, \lambda)}\right) .
$$


Therefore,

$$
\begin{aligned}
f_{\mathfrak{t}^{\lambda} \mathfrak{u}} f_{\mathfrak{u} \mathfrak{t}^{\lambda}} & \equiv \Phi_{\mathfrak{u}, \lambda} \delta^{f-1} \lambda ! F_{\mathfrak{t}^{\lambda}} e_{2 f-1} \cdots e_{n-1} \tilde{\mathfrak{m}}_{\lambda} e_{n-2} \cdots e_{2 f-1} F_{\mathfrak{t}^{\lambda}} \quad\left(\bmod \mathscr{B}_{n}(\delta)^{\triangleright(f, \lambda)}\right) \\
& \equiv \Phi_{\mathfrak{u}, \lambda} \delta^{f-1} \lambda ! f_{\mathfrak{t}^{\lambda} \mathfrak{t}^{\lambda}} \quad\left(\bmod \mathscr{B}_{n}(\delta)^{\triangleright(f, \lambda)}\right),
\end{aligned}
$$

forcing $\left\langle f_{\mathfrak{u}}, f_{u}\right\rangle=e_{\mathfrak{u u}}(n-1) \delta^{f-1} \lambda$ !. By Theorem $[3.18(\mathrm{c})$

$$
\frac{\left\langle f_{\mathfrak{t}}, f_{\mathfrak{t}}\right\rangle}{\delta^{f-1} \mu !}=\frac{1}{\delta^{f-1} \mu !} \frac{\mu_{k}^{2} e_{\mathfrak{t t}}(n-1)}{e_{\mathfrak{u} \mathfrak{u}}(n-1)} e_{\mathfrak{u m}}(n-1) \delta^{f-1} \lambda !=\mu_{k} e_{\mathfrak{t t}}(n-1) .
$$

Finally, we rewrite $e_{\mathfrak{t t}}(n-1)$ via Lemma 3.16a to obtain the formulae, as required.

Proposition 4.9. Suppose $\mathfrak{t} \in \mathscr{T}_{n}^{u d}(\lambda)$ with $(f, \lambda) \in \Lambda_{n}$, and $l(\lambda)=l$. If $\hat{\mathfrak{t}}=\mathfrak{t}^{\mu}$, and $\mathfrak{t}_{n-1}=\mathfrak{t}_{n} \cup p$ with $p=\left(k, \mu_{k}\right) k<l$, define $\mathfrak{u}=\mathfrak{t} s_{n, a+1}$ with $a=2(f-1)+\sum_{j=1}^{k} \mu_{j}$ and $\mathfrak{v}=\left(\mathfrak{u}_{1}, \cdots, \mathfrak{u}_{a+1}\right)$. Define

$$
A=-\delta^{f-1} \mu !\left(\delta+2 \mu_{k}-2 k\right) \prod_{\substack{q \neq p \\ q \in \mathscr{A} \mathscr{R}(\lambda) \geq p}} \frac{c_{\lambda}(p)+c_{\lambda}(q)}{c_{\lambda}(p)-c_{\lambda}(q)} \frac{\prod_{q \in \mathscr{A}(\mu)<p}\left(c_{\mu}(p)-c_{\mu}(q)\right)}{\prod_{r \in \mathscr{R}(\mu)^{<p}}\left(c_{\mu}(p)+c_{\mu}(r)\right)}
$$

Then

$$
\left\langle f_{\mathfrak{t}}, f_{\mathfrak{t}}\right\rangle= \begin{cases}A, & \text { if }\left(k, \lambda_{k}\right) \in R(\lambda) \\ \frac{A}{\delta-2+2 \mu_{k}-2 k}, & \text { if }\left(k, \lambda_{k}\right) \notin R(\lambda)\end{cases}
$$

Proof. By Proposition 4.2, Lemma 4.3, Proposition 4.8 and (4.6), we have

$$
\begin{aligned}
\left\langle f_{\mathfrak{t}}, f_{\mathfrak{t}}\right\rangle & =-\left\langle f_{\mathfrak{u}}, f_{\mathfrak{u}}\right\rangle \frac{1}{\delta+\lambda_{k}-2 k} \frac{\prod_{q \in \mathscr{A}(\mu)<p}\left(c_{\mu}(p)-c_{\mu}(q)\right)}{\prod_{r \in \mathscr{R}(\mu)<p}\left(c_{\mu}(p)+c_{\mu}(r)\right)} \\
\left\langle f_{\mathfrak{u}}, f_{\mathfrak{u}}\right\rangle & =\left\langle f_{\mathfrak{v}}, f_{\mathfrak{v}}\right\rangle \lambda_{k+1} ! \cdots \lambda_{l} ! \\
\left\langle f_{\mathfrak{v}}, f_{\mathfrak{v}}\right\rangle & =\delta^{f-1} \lambda_{1} ! \cdots \lambda_{k} ! \mu_{k}^{2} e_{\mathfrak{v} \mathfrak{v}}(a) .
\end{aligned}
$$

Combining Lemma 3.16 and the above equalities, we obtain Proposition 4.9. Note that $\mu_{k}>1$ in this case.

By the classical branching rule for $\mathscr{B}_{n}(\delta)$ (see [Wen88]),

$$
\Delta(f, \lambda) \downarrow \cong \bigoplus_{\mu \rightarrow \lambda} \Delta(f, \mu) \bigoplus \bigoplus_{\lambda \rightarrow \nu} \Delta(f-1, \nu),
$$

where $\Delta(f, \lambda) \downarrow$ is the restriction of $\Delta(f, \lambda)$ to $\mathscr{B}_{n-1}(\delta)$. We write $(l, \mu) \rightarrow$ $(f, \lambda)$ if $\Delta(l, \mu)$ appears in $\Delta(f, \lambda) \downarrow$. Let $G_{l, \mu}$ be the Gram matrix associated to the cell module $\Delta(l, \mu)$ which is defined by its Jucys-Murphy basis. Let $\operatorname{det} G_{l, \mu}$ be the determinant of $G_{l, \mu}$.

Definition 4.10. Suppose $(f, \lambda) \in \Lambda_{n}$ and $(l, \mu) \in \Lambda_{n-1}$ such that $(l, \mu) \rightarrow$ $(f, \lambda)$. For any $\mathfrak{t} \in \mathscr{T}_{n}^{u d}(\lambda)$ with $\hat{\mathfrak{t}}=\mathfrak{t}^{\mu} \in \mathscr{T}_{n-1}^{u d}(\mu)$, define $\gamma_{\lambda / \mu} \in F$ to be the scalar by declaring that

$$
\gamma_{\lambda / \mu}=\frac{\left\langle f_{\mathfrak{t}}, f_{\mathfrak{t}}\right\rangle}{\delta^{l} \mu !}
$$


Theorem 4.11 provides $\operatorname{det} G_{f, \lambda}$ only recursively. F. Lübeck wrote a GAP program for this recursive formula. It takes us about two hours to compute $\operatorname{det} G_{f, \lambda}$ for all $(f, \lambda) \in \Lambda_{n}$ with $n \leq 35$ using a personal computer.

Theorem 4.11. Let $\mathscr{B}_{n}(\delta)$ be a Brauer algebra over $\mathbb{Z}[\delta]$. Let $\operatorname{det} G_{f, \lambda}$ be the Gram determinant associated to the cell module $\Delta(f, \lambda)$ of $\mathscr{B}_{n}(\delta)$. Then

$$
\operatorname{det} G_{f, \lambda}=\prod_{(l, \mu) \rightarrow(f, \lambda)} \operatorname{det} G_{l, \mu} \cdot \gamma_{\lambda / \mu}^{\operatorname{dim} \Delta(l, \mu)} \in \mathbb{Z}[\delta] .
$$

Furthermore, each scalar $\gamma_{\lambda / \mu}$ can be computed explicitly by Proposition $4.5,4.8$ and 4.9 .

Proof. We first consider Brauer algebras over $\mathbb{C}(\delta)$, where $\delta$ is an indeterminate. Obviously, $\mathbb{C}(\delta)$ satisfies the assumption 3.1. In order to use the seminormal representation constructed in [AMR06, we consider $\mathscr{B}_{n}(\delta)$ over the complex field. Note that $\mathbb{R} \subset \mathbb{C}$. By AMR06, 5.4a], Lemma 3.16(a) holds for $\mathscr{B}_{n}(\delta)$ over $\mathbb{C}$ for infinite many $\delta$. Using the fundamental theorem of algebra, we obtain the result over $\mathbb{C}(\delta)$ where $\delta$ is an indeterminate. Therefore, we can use previous results in this section. Note that the Gram matrix $\tilde{G}_{f, \lambda}$ which is defined via orthogonal basis of $\Delta(f, \lambda)$ is a diagonal matrix. Each diagonal is of the form $\left\langle f_{\mathfrak{t}}, f_{\mathfrak{t}}\right\rangle, \mathfrak{t} \in \mathscr{T}_{n}^{u d}(\lambda)$. Therefore, $\operatorname{det} \tilde{G}_{f, \lambda}=\prod_{\mathfrak{t} \in \mathscr{T}_{n}^{u d}(\lambda)}\left\langle f_{\mathfrak{t}}, f_{\mathfrak{t}}\right\rangle$. By Proposition 4.2 and Corollary 3.7 ,

$$
\operatorname{det} G_{f, \lambda}=\operatorname{det} \tilde{G}_{f, \lambda}=\prod_{(l, \mu) \rightarrow(f, \lambda)} \operatorname{det} G_{l, \mu} \cdot \gamma_{\lambda / \mu}^{\operatorname{dim} \Delta(l, \mu)} .
$$

Since the Jucys-Murphy basis of $\Delta(f, \lambda)$ is defined over $\mathbb{Z}[\delta]$, the Gram matrices associated to $\Delta(f, \lambda)$ which are defined over $\mathbb{Z}[\delta]$ and $\mathbb{C}(\delta)$ are same. We have $\operatorname{det} G_{f, \lambda} \in \mathbb{Z}[\delta]$ as required.

The Gram determinant given in Theorem 4.11 is in $\mathbb{Z}[\delta]$. Note that the Brauer algebra $\mathscr{B}_{n}(\delta)_{F}$ over an arbitrary field $F$ is isomorphic to $\mathscr{B}_{n}(\delta)_{\mathbb{Z}[\delta]} \otimes_{\mathbb{Z}[\delta]} F$. Therefore, one can get the formula for the Gram determinant over an arbitrary field $F$ by specialisation.

We can give a second proof of Theorem 2.8 via Theorem 4.11, We will not give the details here. Instead, we will prove a result for Birman-MurakamiWenzl algebra in [RS06b], which is similar to Theorem 4.11, Via such a formula, we give a necessary and sufficient condition for Birman-MurakamiWenzl algebras being semisimple over an arbitrary field. Certain sufficient conditions for the semisimplicity of Birman-Murakami-Wenzl algebras over complex field were obtained by Wenzl in Wen90.

Example 4.12. The Gram determinant associated to the cell module $\Delta(1, \lambda)$ for $\mathscr{B}_{4}(\delta)$ with $\lambda=(2) \vdash 2$.

There are six elements in $\mathscr{T}_{4}^{u d}(\lambda)$ as follows. 


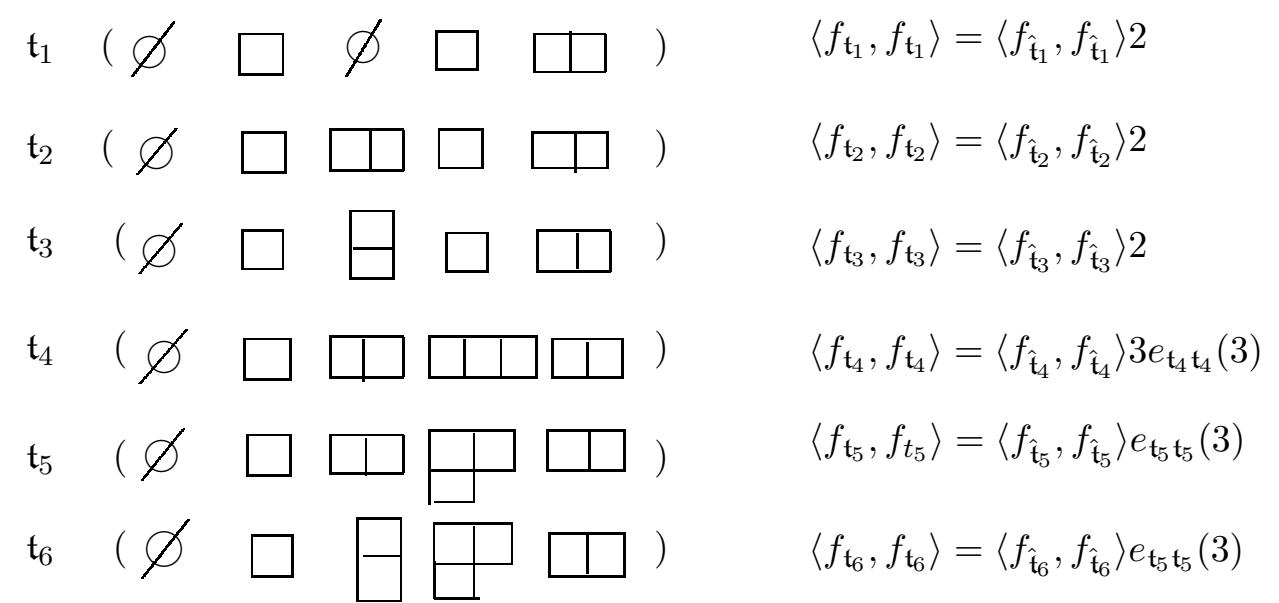

Define $\mathfrak{m}_{\mathfrak{t}_{1}}=e_{1}\left(1+s_{3}\right), \mathfrak{m}_{\mathfrak{t}_{2}}=e_{1}\left(1+s_{3}\right) s_{2}\left(1+s_{1}\right), \mathfrak{m}_{\mathfrak{t}_{3}}=e_{1}\left(1+s_{3}\right) s_{2} s_{1}$, $\mathfrak{m}_{\mathfrak{t}_{4}}=e_{1}\left(1+s_{3}\right) s_{2} s_{3} s_{1} s_{2}\left(1+s_{2}+s_{2} s_{1}\right), \mathfrak{m}_{\mathfrak{t}_{5}}=e_{1}\left(1+s_{3}\right) s_{2} s_{3} s_{1} s_{2}$ and $\mathfrak{m}_{\mathfrak{t}_{6}}=$ $e_{1}\left(1+s_{3}\right) s_{2} s_{3} s_{1}$. Then $\left\{\mathfrak{m}_{\mathfrak{t}_{i}}\left(\bmod \mathscr{B}_{4}(\delta)^{\triangleright \lambda}\right) \mid 1 \leq i \leq 6\right\}$ is the JucysMurphy base of $\Delta(1,(2))$. The corresponding Gram matrix $G_{1,(2)}$ is given as follows:

$$
G_{1,(2)}=\left(\begin{array}{cccccc}
2 \delta & 4 & 2 & 4 & 0 & 2 \\
4 & 4 \delta+4 & 2 \delta+2 & 8 & 4 & 2 \\
2 & 2 \delta+2 & 2 \delta & 4 & 2 & 2 \\
4 & 8 & 4 & 6 \delta+12 & 2 \delta+4 & 2 \delta+4 \\
0 & 4 & 2 & 2 \delta+4 & 2 \delta & 2 \\
2 & 2 & 2 & 2 \delta+4 & 2 & 2 \delta
\end{array}\right)
$$

Therefore, $\operatorname{det} G_{1,(2)}=64 \delta^{3}(\delta-2)^{2}(\delta+4)$. On the other hand, by the formulae on $\gamma_{\lambda / \mu}$, we obtain $e_{\mathfrak{t}_{4} \mathfrak{t}_{4}}(3)=\frac{\delta(\delta+4)}{3(\delta+2)}$ and $e_{\mathfrak{t}_{5} \mathfrak{t}_{5}}(3)=\frac{2 \delta(\delta-2)}{3(\delta-1)}$. By Theorem 4.11 again, we recover the formula for $\operatorname{det} G_{1,(2)}$.

\section{REFERENCES}

[AMR06] S. Ariki, A. Mathas and H. Rui, "Nazarov-Wenzl algebras", Nagoya Math. $J$., Special volume in honor of G. Lusztig's 60-th birthday, 182, 47-134, (2006).

[BW89] J. S. BiRMAN and H. WenzL, "Braids, link polynomials and a new algebra", Trans. A.M.S 313, 249-273, (1989).

[Bra37] R. Brauer, "On algebras which are connected with the semisimple continuous groups", Ann. Math. 38, 857-872, (1937).

[Bro56] W. P. Brown, "The semisimplicity of $\omega_{f}^{n}$ ", Ann. Math. (2) 63, 324$335,(1956)$.

[DJ86] R.Dipper, AND G. JAMEs, "Representations of the Hecke algebras of general linear groups", Proc. L.M.S (3) 52, 20-52, (1986).

[DWH99] W. F. Doran, IV, D. B. Wales and P. J. Hanlon, "On the semisimplicity of the Brauer centralizer algebras", J. Algebra 211, 647-685, (1999).

[Eny04] J. ENyAng, "Cellular bases for the Brauer and Birman-Murakami-Wenzl algebras", J. Algebra 281, 413-449, (2004).

[Eny05] J. ENYANG, "Specht modules and semisimplicitry criteria for Brauer and Birman-Murakami-Wenzl algebras", J. Algebraic Combin., to appear. 
[FG95] S. Fishel and I. Grojnowski, "Canonical bases for the Brauer centralizer algebra", Math. Res. Lett. 2, 15-26, (1995).

[GL96] J. J. Graham and G. I. Lehrer, "Cellular algebras", Invent. Math. 123, 1-34, (1996).

[HR95] T. HALvERSON and A. RAM, "Characters of algebras containing a Jones basic construction: the Temperley-Lieb, Okasa, Brauer, and Birman-Wenzl algebras", Adv. Math. 116, 263-321, (1995).

[HW89a] P. HANLON and D. WALES, "Eigenvalues connected with Brauer's centralizer algebras", J. Algebra 121, 446-476, (1989).

[HW89b] , "On the decomposition of Brauer's centralizer algebra", J. Algebra 121, 409-445, (1989).

[JM00] G. D. JAMES and A. MAThas , "The Jantzen sum formula for cyclotomic q-Schur algebras,", Trans. A.M.S. 352, 5381-5404, (2000).

[Jon94] V. F. R. Jones, "A quotient of the affine Hecke algebra in the Brauer algebra", 'Enseign. Math. 40, 313-344, (1994).

[LR97] R. LEDUC and A. RAM, "A ribbon Hopf algebra approach to the irreducible representations of centralizer algebras: the Brauer, Birman-Wenzl, and type A Iwahori-Hecke algebras", Adv. Math. 125, 1-94, (1997).

[Mat99] A. Mathas, Hecke algebras and Schur algebras of the symmetric group, Univ. Lecture Notes, 15, Amer. Math. Soc., 1999.

[Mat04] "Matrix units and generic degrees for the Ariki-Koike algebras", J. Algebra 281, 695-730, (2004).

[Mat06] _ "Seminormal forms and Gram determinants for cellular algebras", preprint, 2006

[MW00] H.R. Morton and A.J. Wassermann , "A basis for the Birman-Wenzl algebra", preprint, 2000.

[Mur83] G.E MurPhy, "The idempotents of the symmetric groups and Nakayama comjecture", J. Algebra, 81, 258-265, (1983).

[Naz96] M. NAzARov, "Young's orthogonal form for Brauer's centralizer algebra", J. Algebra 182, 664-693, (1996).

[Ram95] A. RAm, "Characters of Brauer's centralizer algebras", Pacific J. Math. 169, 173-200, (1995).

[Rui05] H. Rui, "A criterion on semisimple Brauer algebra", J. Comb. Theory, Ser. A 111, 78-88, (2005).

[RS06a] H. Rui AND M. Si, "A criterion on semisimple Brauer algebra, II", J. Comb. Theory, Ser. A, 113, 1199-1203, (2006)

[RS06b] — "Gram determinants and semisimple criteria for Birman-Wenzl algebras, ", Arxiv preprint math.QA/0607266, (2006).

[RX05] H. Rui and J. Xu, "On the semisimplicity of cyclotomic Brauer algebras, II", J. Algebra, to appear.

[RY04] H. RuI and W. YU, "On the semisimplicity of cyclotomic Brauer algebras", J. Algebra 277, 187-221, (2004).

[Ter01] I. TERADA, "Brauer diagrams, updown tableaux and nilpotent matrices", $J$. Algebraic Combin. 14, 229-267, (2001).

[Wen88] H. WenZL, "On the structure of Brauer's centralizer algebras", Ann. Math. 128, 173-193, (1988).

[Wen90] H. WenzL, "Quantum groups and subfactors of type B,C, and D", Comm. Math. Phys. 133 (1988), 383-432.

[Wey97] H. WEYL, "The classical groups", Princeton Landmarks in Mathematics, Princeton University Press, Princeton, NJ, Their invariants and representations, Fifteenth printing, Princeton Paperbacks, 1997.

H.R. Department of Mathematics, East China Normal University, Shanghai, 200062, CHINA

E-mail address: hbrui@math.ecnu.edu.cn 
M. S. Department of Mathematics, East China Normal University, ShangHAI, 200062, CHINA

E-mail address: 52050601011@student.ecnu.edu.cn 